Check for updates

Cite this: Mater. Adv., 2021, 2, 3441

Received 28th February 2021 Accepted 29th March 2021

DOI: $10.1039 / \mathrm{d} 1 \mathrm{ma} 00174 \mathrm{~d}$

rsc.li/materials-advances

\title{
Biosafety of micro/nanomotors towards medical application
}

\author{
Kexin $\mathrm{Hou}^{{ }^{a}}$ Yandong Zhang, ${ }^{a}$ Meili Bao, ${ }^{a}$ Yanhao Liu, ${ }^{a}$ Jinmeng Wang, ${ }^{a}$ Chao Xin, ${ }^{a}$ \\ Zengyan Wei, ${ }^{a}$ Hua Zhang, ${ }^{a}$ Zhiguang Wu ${ }^{1}{ }^{b}$ and Zhenyu Wang ${ }^{* a}$

\begin{abstract}
Micro/nanomotors (MNMs) have attracted tremendous interest of researchers owing to their ability to mimic the function of natural microorganisms to complete tasks in biomedicine and other fields. However, in order to realize their practical use in the biomedical field, MNMs are required to meet the biosafety standard, which has not been systematically reviewed. The most important thing of concern for biosafety is to fabricate MNMs with good biodegradability, biocompatibility and biosafe propulsion. We reviewed the state-of-the-art of biosafe MNMs, focusing on the biodegradability, biocompatibility, biosafe propulsion and biosafety assessments of MNMs. The challenges and opportunities of biosafe MNMs for biomedical application are discussed.
\end{abstract}

\section{Introduction}

With the rise in the exploration of the microcosm, the goal to achieve complex tasks on demand in biomedical and environmental fields has led to the development of micro/nanomotors (MNMs). Various artificial MNMs have been fabricated sophisticatedly in an inherent asymmetric nature, with physically or chemically responsive segments. The unique structure of artificial MNMs has enabled the motors to have motion, propulsion, or collective chemotaxis behavior. MNMs are desired for achieving tasks in a controlled and dynamic fashion, such as drug delivery, cancer diagnostics, manipulation of cells, detoxification, nanosurgery, imaging guided therapy, sensing and environmental remediation..$^{1-5}$ As a persistent endeavor, the application of MNMs in the biomedical field is the most promising but challenging. For application in the biomedical field, MNMs are expected to be biosafe, highly efficient, flexible and controllable. With the indepth research, the progress of intelligent design, functionality and controllability of MNMs has been fully studied and reviewed during the past few years. There has been increasing interest in exploring a wide range of biosafe MNMs for various medical applications. However, the highly desirable biosafety of artificial MNMs has not been systematically studied.

For biomedical application in practice, the biosafety issues of artificial MNMs including the building blocks, propulsion

\footnotetext{
${ }^{a}$ School of Chemistry and Chemical Engineering, Harbin Institute of Technology, Xidazhi Street, Nangang District, 150001 Harbin, No. 92, Heilongjiang Province, China. E-mail: wzy219001@163.com; Fax: +86045186282909; Tel: +86045186282909

${ }^{b}$ Key Laboratory of Micro-systems and Micro-structures Manufacturing (Ministry of Education), Harbin Institute of Technology, 150080 Harbin, China
}

mechanisms and assessment method are of great significance to ensure the feasibility. ${ }^{6}$ While many kinds of materials have been explored to fabricate MNMs, some of the materials which are non-degradable and non-biocompatible would be difficult to implement for in vivo application. The use of biosafe materials and propulsion may contribute to solving the bottleneck problems of artificial MNMs in practical applications and translate proof-ofconcept into next generation applications.

Here, we focus on the biosafety issues of artificial MNMs including biodegradability, biocompatibility, biosafe propulsion as well as biosafety assessments. Biodegradability and biocompatibility problems should be carefully considered during biomedical MNMs' design because these MNMs are expected to be expelled out and pose no health hazard to the body. In addition, propulsion mechanisms of MNMs need to be benign to the biotic environment. The integration of biosafe materials and propulsion infuses new energy in the fabrication of functional MNMs. Biodistribution and toxicity in vitro and in vivo are explored to assess the biosafety issues. For the final part, future perspectives and challenges of biosafe MNMs are discussed.

\section{Biodegradability}

Some intravenously given artificial MNMs would interact with the constituents of the blood and trigger immune response. $6,7,16,40,85,88$ Degradable artificial MNMs are expected to be easily absorbed by the organism when they have fulfilled the biological functions and to reduce/avoid immune response caused by traditional materials or medicine, while the intrinsic qualities of MNMs such as small size and large surface area enable them to easily interact with the constituents of blood and induce immune toxicity. There is 
a growing demand to develop various materials in order to reduce immune toxicity, improve medical outcomes and promote practical medical applications of MNMs.

\subsection{Biodegradation mechanisms}

Specifically, the biodegradation mechanisms of some common materials such as natural and synthetic polymers involve cellular activities and enzymatic reactions, while the biodegradation mechanisms of biodegradable metals and carbonate systems involve chemical reactions in the physiological environment. ${ }^{7}$ The biodegradation of materials starts immediately after the materials enter the body and it causes physicochemical changes including changes in size, weight, surface topography, mechanical strength and density. ${ }^{8}$ The degradation kinetics of biodegradable materials is determined by both the physicochemical properties of the materials and physiological states within living creatures. Controlling the degradation kinetics of biodegradable materials is crucial for their successful medical applications. Under ideal conditions, the materials first maintain their integrity, perform their role and function with no obvious mass loss and deformation. After the biological function is completed, the degradation rate of the material is accelerated, so as to realize rapid mass loss until the complete degradation of the material.

Biomaterials usually elicit a host response once they enter the body and induce inflammation or proliferation. ${ }^{17,84,86,87,96}$ The events relating to host response to biodegradable materials are considered as stage-dependent. Some acute host response is caused by protein adsorption onto the surface of materials when the materials enter the body. The degree of host response is related to the level of biodegradation kinetics and biodegradation products. Host response also influences the biodegradation process and the performance of materials, causing structural and morphological development. The degradation of materials may also be accompanied by some changes in the immediate vicinity of the materials due to the production of intermediate degradation products or soluble degradation products, and these changes also affect host response.

\subsection{Natural degradable polymers}

Natural degradable polymers including proteins (urease and bovine serum albumin), polypeptides (poly-L-lysine) and polysaccharides (alginate and chitosan) have drawn much attention and been widely used in the fabrication of MNMs for biomedical applications. Owing to the resemblance of natural degradable polymers to biological macromolecules, natural polymers often show promising biological activities, possess cell surface binding ligands, are easier to be degraded by cell-mediated enzymes and are susceptible to natural remodeling. Natural biodegradable polymers are mainly degraded through enzymatic degradation, oxidation and phagocytosis. After entering the body, the MNMs fabricated using natural polymers are less likely to induce adverse foreign-body reaction.

Alginate (ALG) and chitosan (CHI) as natural polysaccharides, have controllable biodegradability, excellent biocompatibility and viscosity. Yang et al. ${ }^{9}$ had combined negatively charged ALG and positively charged $\mathrm{CHI}$ to form biodegradable nanotubes via the layer-by-layer (LbL) assembly technique. The assembled nanotubes were degraded under the action of enzyme lipase as they were immersed in pancreatin solution. This indicated that the ALG/CHI nanotubes had good biodegradable feature. On the basis of this research, Wu et al. ${ }^{10}$ assembled platinum nanoparticles (Pt NPs) within the inner surface of ALG/CHI nanotubes to catalyze the decomposition of hydrogen peroxide $\left(\mathrm{H}_{2} \mathrm{O}_{2}\right)$ into oxygen and water. The oxygen bubbles were released from the opening of the nanorockets and in turn pushed the vehicle along. In order to achieve controllable locomotion of the biodegradable nanorockets, $\mathrm{Fe}_{3} \mathrm{O}_{4}$ nanoparticles were modified on the surface of the tubular nanorockets.

The versatility of the LbL technique allows the frame structure properties of polyelectrolyte microcapsules to be modulated by various functional components. Xuan et al. ${ }^{11}$ reported the gold nanorod (GNR) functionalized Janus ALG/CHI polyelectrolyte microcapsule micromotor. Because of the photothermal effect of GNRs, the asymmetric structure could be triggered using nearinfrared (NIR) light and realize the unidirectional movement. Hu et $a .^{12}$ fabricated a biodegradable star-shaped hydrogel MNM by using poly(vinyl alcohol) and ALG; ALG as the main component of the vehicle bestowed the MNM with good biodegradability, as it was degraded by amylase. By adding magnetic nanoparticles, the star-shaped hydrogel can achieve controlled motion in various biofluids under an external magnetic field.

Natural polymers bovine serum albumin (BSA) and poly-Llysine (PLL) have been used as building units for the fabrication of protein and polypeptide based rockets by $\mathrm{Wu}$ et $a{ }^{13}{ }^{13}$ As shown in Fig. 1A, a heat-sensitive gelation hydrogel was also integrated into the microtube along with gold nanoparticles (Au NPs), doxorubicin (DOX) and catalase. The $\mathrm{H}_{2} \mathrm{O}_{2}$ fuel was expected to diffuse from the surrounding liquid medium to catalase and be catalyzed into water and oxygen bubbles which were expelled via the opening of the microtubes to propel the rockets. Upon NIR illumination, the gelatin hydrogel melted and released the encapsulated DOX under the photo-thermal effect of Au NPs. The good biodegradability of the rockets was evaluated by incubating the rocket with $\alpha$-chymotrypsin.

The functional unit protein is the common component to be integrated into microtubes or capsules, affording them responsiveness to various stimuli. Sugai et al. ${ }^{14}$ presented "all-protein" microtube motors with a urease interior surface which were fabricated via template-assisted LbL assembly and Avi-biotin interaction (as shown in Fig. 1B). Such protein microtubes having nontoxic urea fuel can be propelled by the Ure reaction (urea $\rightarrow \mathrm{NH}_{3}+\mathrm{CO}_{2}$ ). The protein based MNMs having good biodegradability could be broken down in acidic water and completely digested by proteases. As depicted in Fig. 1C, PenaFrancesch et al. ${ }^{15}$ reported biodegradable and multifunctional self-propelled protein-based MNMs to overcome the material and performance limitations (low efficiency, toxicity and lack of locomotion direction control) of self-propelled MNMs based on Marangoni propulsive forces. Squid ring teeth (SRT) proteins and hexafluoroisopropanol (HFIP) were applied to fabricate the chemical motors. Through regulating the nanostructure of SRT proteins and the interaction with HFIP fuel, the self-propelled 


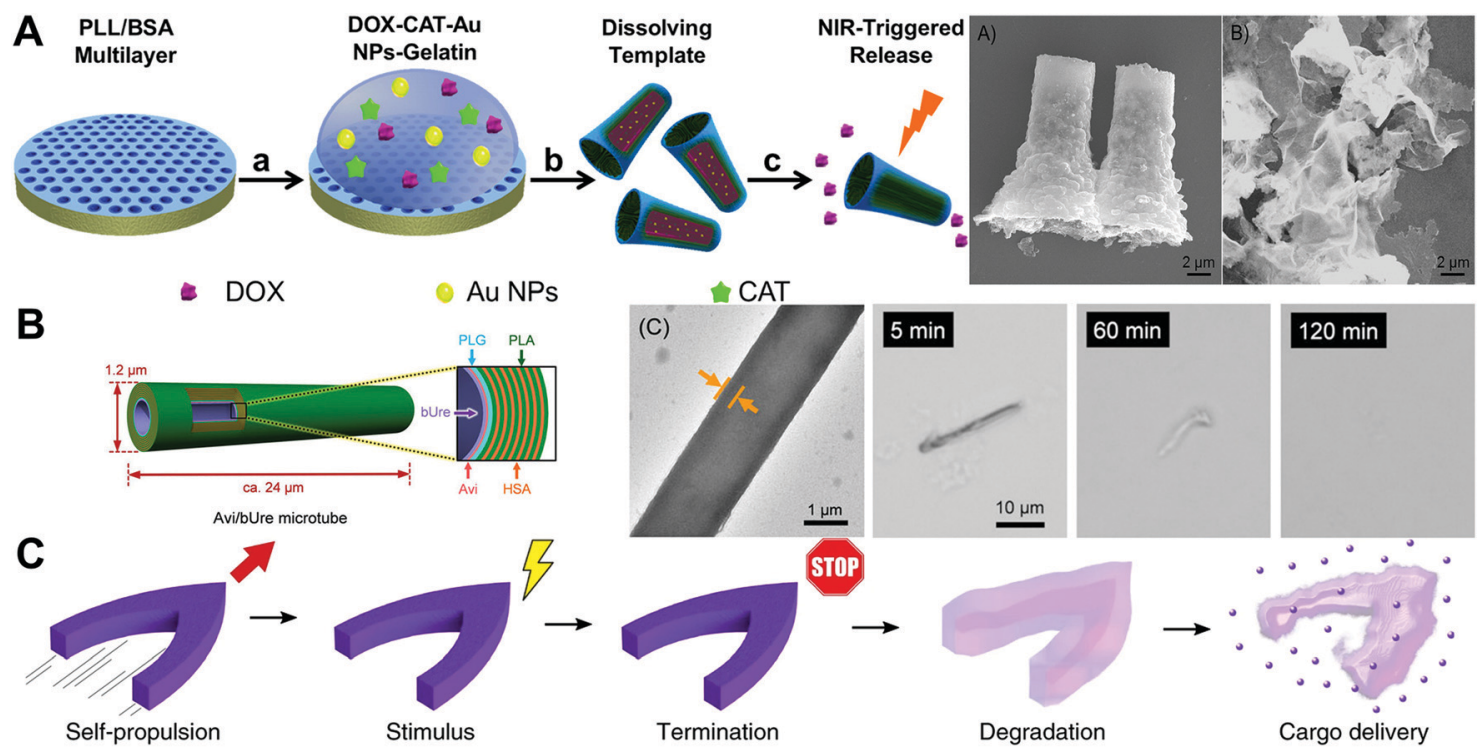

Fig. 1 Natural degradable polymer based MNMs. (A) Scheme illustrating the fabrication and light-triggered drug release process of protein-based rockets and SEM images of these rockets before and after incubating with $\alpha$-chymotrypsin solution. (B) Schematic illustration of the Avi/bUre microtube, TEM image and morphology changes of the prepared motors over time in Pronase solution at $37^{\circ} \mathrm{C}$. (C) Schematic illustration of protein motor ondemand termination and degradation for cargo delivery. (A) Reproduced with permission. ${ }^{13}$ Copyright 2015, American Chemical Society. (B) Reproduced with permission. ${ }^{14}$ Copyright 2019, Wiley-VCH. (C) Reproduced with permission. ${ }^{15}$ Copyright 2019, Springer Nature.

protein-based MNMs outperformed previous chemical motors by several orders of magnitude: programmable locomotion, on-demand termination and degradation. In addition, the protein matrix could be naturally degraded and release the encapsulated drugs in an acidic environment. The contributions introduced in this section offer great potential toward the development of biodegradable MNMs by choosing natural polymers which resemble biological macromolecules for medical applications.

\subsection{Synthetic degradable polymers}

Unlike natural degradable polymers, synthetic polymers demonstrate a good remanufacturability with controlled composition, allowing one to adjust the physicochemical properties of materials and improve the functions of MNMs. Additionally, the degradation kinetics of synthetic polymers could be tuned by modulating the physicochemical properties of the polymers, blending, copolymerization or surface modifications. Since the synthesis of the first degradable suture using the synthetic polymer polyglycolide in the 1960s, various degradable synthetic polymers such as polycaprolactone (PCL), polyglycolide (PGA), polylactide (PLA) and polyurethanes have been produced. With the in-depth research of MNMs during the past decade, a growing number of researchers began to pay attention to the application of degradable synthetic polymers in MNMs. Synthetic polymers degrade via hydrolysis, oxidation and enzymatic degradation. During the course of hydrolysis of the ester bond in the polymer chain, some synthetic polymers including polyesters, polyorthoesters, polycarbonates, polyanhydrides and polyamides were degraded into oligomers or monomers, which could be cleared by the organism. ${ }^{16}$ In particular, the hydrolytic products of some synthetic polymers including PCL, PGA and PLA are small molecules, such as lactic acid, glycolic acid and 6-hydroxyhexanoic acid, and these acidic degradation products could be oxidized in the tricarboxylic acid cycle and provide energy for organisms. ${ }^{16,17}$ Synthetic polymers could also be degraded gradually by some enzymes that are present in the blood or secreted by immune cells which could break apart the chemical bonds in polymer chains, or degraded through phagocytosis by macrophages or other cells. Besides, synthetic polymers could be depolymerized by oxygen free radicals which were released from some inflammatory cells via oxidation. ${ }^{18}$

As shown in Fig. 2B, the magnetic composite non-toxic and biodegradable photocrosslinkable hydrogel gelatin methacryloyl (GelMA) had been applied to manufacture 3D printed soft helical MNMs. ${ }^{19}$ Based on the intrinsic soft nature of GelMA, the fabricated helical MNMs can keep their forward velocity as the rotating frequency increases, suggesting a unique self-adaptive behavior. Full GelMA MNM microstructures were proteolytically biodegradable by collagenases in the extracellular matrix. GelMA MNMs, the GelMA precursor and the degradation products of magnetic-composite GelMA MNMs were all nontoxic.

There are more and more research studies on the characters and applications of PCL, which is an FDA-approved biodegradable synthetic polymer. A kind of biodegradable MNM was fabricated by a scalable self-assembly method using PCL single crystals and silver nanoparticles as the framework. ${ }^{20}$ Due to the nature of polymer single crystals and nanoparticles, the reported MNMs exhibited multiple functions such as molecular detection, autonomous movement, and sustained and controlled drug release. A kind of "all-polymer" MNM containing PCL single crystals as the main part and catalase as the engine was reported by Liu et al. ${ }^{21}$ With catalase immobilized onto the surface of PCL crystals by chemical reactions, this all-polymer MNM performed selfpropelled motion where catalase functioned as an engine to catalytically decompose $\mathrm{H}_{2} \mathrm{O}_{2}$ to water and oxygen. 

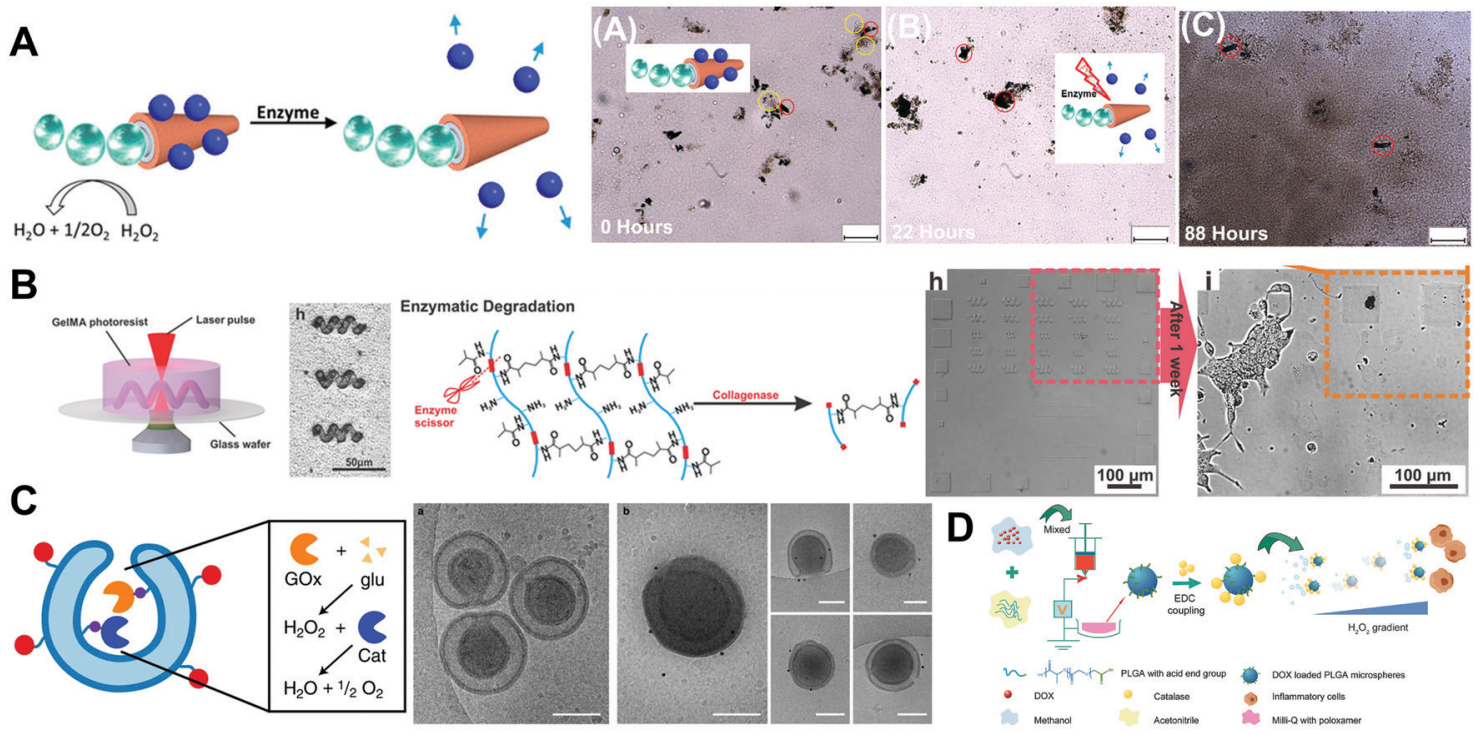

Fig. 2 Synthetic degradable polymer based MNMs. (A) Schematic illustration of the fabrication process and enzymatically triggered disintegration of micromotors, consecutive image frames of enzymatically triggered disassembly of these micromotors, nanosphere release, and degradation of the whole transport system. (B) Schematic illustration of fabrication of biodegradable GelMA helical micromotors. Optical image of helical micromotors decorated with magnetic nanoparticles, illustration of enzymatic degradation of GelMA and images of partially degraded GelMA helical microstructures after incubation with $\mathrm{HaCaT}$ cells. (C) Schematic illustration of polymeric nanomotors with spatial control over the catalyst and Cryo-TEM pictures of poly(ethylene glycol)- $b$-poly $(\mathrm{D}, \mathrm{L}$-lactide) stomatocytes after reduction with TCEP beads and subsequent reaction with NHS functionalized gold nanoparticles. (D) Schematic diagram for the preparation of motored PLGA particles. (A) Reproduced with permission. ${ }^{23}$ Copyright 2019 , American Chemical Society. (B) Reproduced with permission. ${ }^{19}$ Copyright 2018, Wiley-VCH. (C) Reproduced with permission. ${ }^{25}$ Copyright 2019 , Springer Nature. (D) Reproduced with permission. ${ }^{26}$ Copyright 2019, Wiley-VCH.

Spindle-like MNMs consisting of PCL and an anionic surfactant were fabricated using a one-step electrospinning method. ${ }^{22}$ The spindle-like MNMs were self-propelled based on Marangoni propulsive forces on the surface of water. The release of the anionic surfactant, sodium 1-dodecanesulfonate, from the spindle-like MNMs induced propulsive Marangoni forces. As indicated in Fig. 2A, Kroupa et al. ${ }^{23}$ presented the novel concept of a degradable mothership based on PCL polymers capable of delivering cargo-carrying nanospheres to target sites. The main part of the polymer mothership (PCL-SH) was synthesized through green ring-opening polymerization. PCL-SH was then coated with a compact platinum $(\mathrm{Pt})$ layer to make sheet-like and tubular MNMs after sonication. A biodegradable self-assembled polymeric stomatocyte MNM for drug delivery was built using a mixture of poly(ethylene glycol)-b-poly(e-caprolactone) and the well-documented vesicle former poly(ethylene glycol)-b-polystyrene. ${ }^{24}$ DOX was encapsulated in the lumen of the microstructure while Pt NPs acting as the engine were loaded in the cavity of the polymeric stomatocyte structure. A high proportion of PCL mixed in the structure of the hybrid vehicle resulted in the formation of PCL domains. During the degradation of PCL, the membrane of the stomatocyte would collapse and release the encapsulated drugs after the MNMs were taken by tumor cells. Owing to the biodegradability of PCL, all the above mentioned MNMs based on PCL could be disassembled into tiny pieces and trigger drug release with the action of enzymes. As indicated in Fig. 2C, a multivalent design of biodegradable polymeric MNMs was proposed for the preparation of stomatocytes. ${ }^{25}$ Stomatocytes were made from biodegradable synthetic polymers poly(ethylene glycol)- $b$-poly(D,L-lactide) including azide terminated polymers as functional handles. Two different functional handles on the inner and outer surface provided spatial control over the catalyst. Catalase along with glucose oxidase was loaded inside the cavity to propel the polymeric MNMs in the presence of fuel.

As shown in Fig. 2D, through the functionalized poly(lacticco-glycolic acid) (PLGA) microparticles with catalase, a biodegradable MNM performing autonomous motion was designed to deliver drugs to inflammatory lesions. ${ }^{26}$ PLGA MNMs moved faster with increasing levels of $\mathrm{H}_{2} \mathrm{O}_{2}$, and finally they performed directional movement toward macrophages where $\mathrm{H}_{2} \mathrm{O}_{2}$ was found at elevated levels. The core/shell polymeric microtube motors made of PLGA and polyethylene oxide were fabricated by coelectrospinning and cryosectioning. ${ }^{27}$ The use of synthetic biodegradable polymers as the backbone of microtubes allowed for taking advantages of their phase changes, resulting in the shape reconfiguration of microtubes when exposed to ultrasound. This approach allowed for converting the microtubes into picoliter-scale containers. MNMs based on metal was not applicable in biological settings. Toebes et $\mathrm{al}^{28}$ reported the first biodegradable polymersome MNMs with a tubular shape. The framework of the polymeric MNMs was made from poly(ethylene glycol)-b-poly(D,L-lactide). Poly(D,L-lactide) as an FDA-approved biodegradable synthetic polymer is a promising candidate for MNM materials. The tubular shaped polymersome MNMs were decorated with catalase to provide propulsion through catalytic decomposition of $\mathrm{H}_{2} \mathrm{O}_{2}$ into oxygen and water.

The use of various synthetic polymers offers great potential toward the development of biodegradable and intelligent drug 
delivery systems through controlling the composition and physicochemical properties of synthetic polymers for the construction of MNMs. The assembled MNMs are usually sensitive to changes in the environment, including chemical, physical or biological stimuli.

\subsection{Biodegradable metals}

Unlike polymers, metals with better mechanical properties and shorter degradation periods, have attracted more and more attention. Biodegradable metals such as magnesium (Mg), iron (Fe), and zinc ( $\mathrm{Zn})$ and their alloys are defined as metals expected to degrade via chemical reactions in physiological environments. Besides, metal debris after the chemical reaction can also be phagocytosed. Biodegradable metals react with water through a range of anodic and cathodic reactions to generate hydrogen bubbles and hydroxides, and can thus be regarded as powering MNMs. Furthermore, $\mathrm{Mg}, \mathrm{Fe}$ and $\mathrm{Zn}$ are metal trace elements which are essential to maintain health and body functions. $\mathrm{Mg}$ has a high daily allowable dose of approximately $400 \mathrm{mg}$, while $\mathrm{Fe}$ and $\mathrm{Zn}$ as micronutrients have a daily allowable dose of less than $100 \mathrm{mg} \cdot{ }^{29-31}$ Biodegradable metals possess a broad development prospect as chemical propellents due to their biodegradability, biocompatibility and transient nature in organisms.

Alkali earth metals capable of reacting with water to form hydrogen bubbles could be regarded as constituents for activating MNMs. However, alkali earth metals including $\mathrm{Na}$ and Ca cannot be electrodeposited; besides their reactions with water are too violent to be controlled due to their intrinsic chemistry nature. One of the earliest examples of water-driven bubble-propelled MNMs using biodegradable metals was proposed by Gao et al. ${ }^{32}$ The aluminum-gallium (Al-Ga) binary alloy microsphere was propelled by hydrogen bubbles through the reaction of Al-alloy with water. Compared with reactive alkali metals, Al-alloy based MNMs offered better stability and lower costs. However, the fabricated Al-alloy based MNMs were toxic because $\mathrm{Al}$ and $\mathrm{Ga}$ species could be continuously released into the microenvironment. Transition metals including $\mathrm{Fe}$ and $\mathrm{Zn}$ are considered as alternative chemical propellents because of their transient nature, biodegradability as well as biocompatibility with organisms. Gao et al. ${ }^{33}$ had fabricated poly(3,4-ethylenedioxythio-phene)/Zn MNMs through a membrane templating process and examined their behaviors along with functionalities under in vivo conditions for the first time. Zn-based MNMs had distinct advantages in biomedical applications especially for gastric drug delivery due to their unique characteristics such as acid-powered propulsion, nontoxic self-destruction and autonomous delivery. The Zn part of MNMs was dissolved in the acidic environment, then the motors were self-destroyed, releasing the loaded cargoes, and left nothing toxic behind. As shown in Fig. 3B, Zhou et al. ${ }^{34}$ had presented a drugloading biodegradable microrocket with a core of $\mathrm{Zn}$, a thin $\mathrm{Fe}$ intermediate layer and an outer poly(aspartic acid) layer. The galvanic corrosion of $\mathrm{Zn}$ particles promoted by the Fe intermediate layer could propel the microrockets via producing hydrogen bubbles in the gastric acid environment. In addition, the Fe intermediate layer also facilitated magnetic guidance of microrockets. As all materials used in the microrockets were biodegradable under the action of gastric fluid or protease, the motors could be self-destroyed with non-harmful residue in vivo.

However, transition metals usually corrode slowly in neutral water resulting in inefficient bubble-recoiling propulsion. It is worth mentioning that $\mathrm{Mg}$ has been considered as a promising candidate material for its moderate reaction rate with water, biodegradability as well as biocompatibility with organisms. ${ }^{35}$

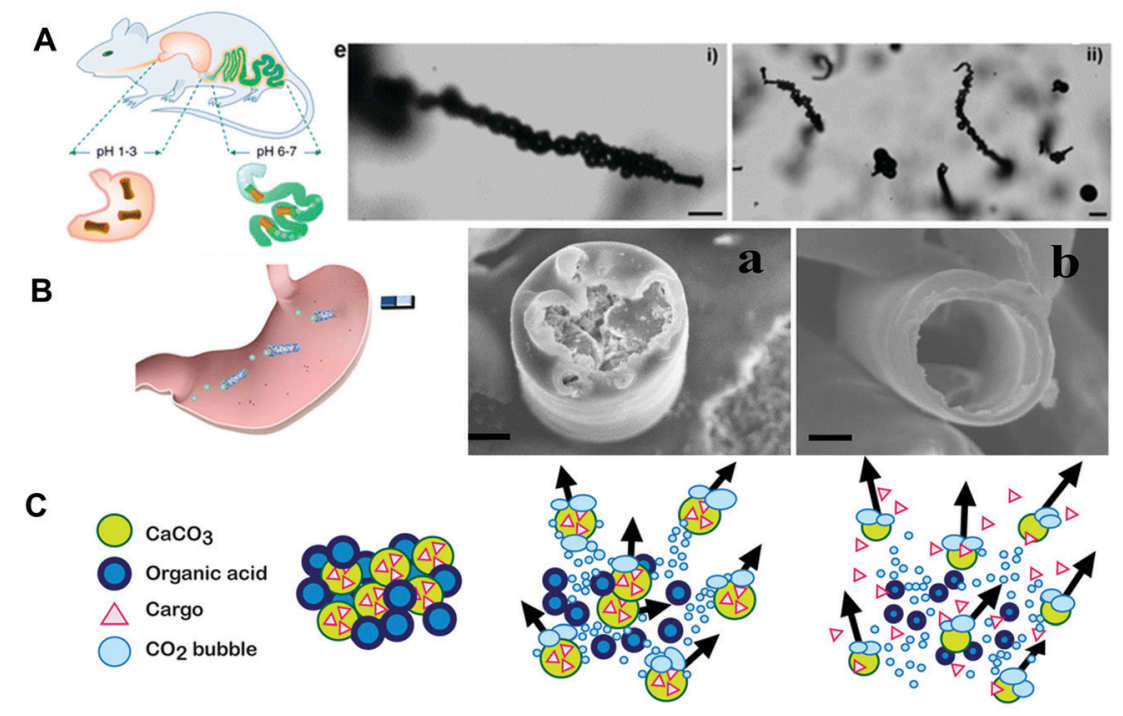

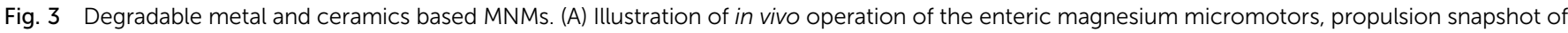

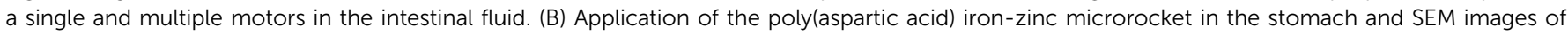

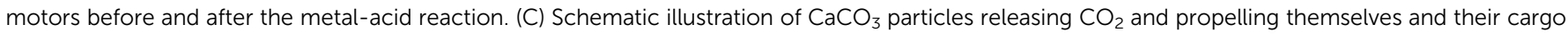

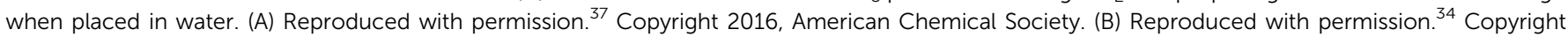

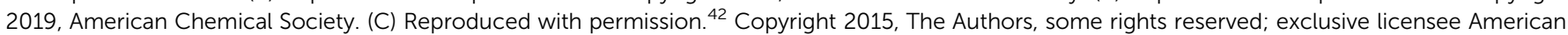
Association for the Advancement of Science. 
Chen et $a l .{ }^{36}$ demonstrated the propulsion and degradation characteristics of different Janus MNMs based on $\mathrm{Zn} / \mathrm{Fe}$, $\mathrm{Zn}, \mathrm{Mg} / \mathrm{ZnO}$ and $\mathrm{Mg} / \mathrm{Si}$ materials. The self-destroyed MNMs dissolved harmlessly in the physiological environment after accomplishing a specific task. The degradation properties of these MNMs depended on the different corrosion rates of the constituent transient materials. As shown in Fig. 3A, a desirable gastrointestinal tract delivery system was developed based on a drug-loaded Mg MNM body with enteric polymer coating. ${ }^{37}$ The enteric coating could protect the drugs en route and dissolved at the target site because it was stable in the acidic gastric fluid but soluble in the intestinal fluid. Upon arrival at the target site, the MNMs were exposed to the fuel, leading to the activation of their propulsion and the complete unloading of cargos. An antibacterial approach was proposed which coupled the degradable $\mathrm{Mg}$-based MNMs with bacteria killing properties of CHI. ${ }^{38}$ The self-propelled Janus microspheres containing a Mg-based framework were coated with biodegradable polymers PLGA, ALG and CHI. The highly degradable MNM-based antibacterial approach offered significant improvements in the antibacterial efficiency than static CHI-coated microparticles. Lopez-Ramirez et al. ${ }^{39}$ had proposed a biodegradable, active and autonomous microneedle delivery platform based on the encapsulation of $\mathrm{Mg}$ microparticles within the degradable polymeric microneedle patch, thus avoiding unnecessary external systems for triggering active microneedle delivery.

Biodegradable metal-based micromotors hold great promise in various applications because their fuel - water - is clean, safe and ubiquitous to both environmental ecological system and biological system. Those biodegradable metal-based MNMs are easily degraded in neutral or acidic water, with nontoxic residues from the functionalized components. MNMs mainly consisting of $\mathrm{Mg}$, $\mathrm{Zn}$ or Fe matrices can not only be applied as drug delivery systems, but also as nutritional supplements due to their inherent properties.

\subsection{Carbonate systems}

Some carbonate systems such as calcium carbonate $\left(\mathrm{CaCO}_{3}\right)$ can degrade in vivo, while their degradation behaviors depend on the chemical composition and microstructure of the material. ${ }^{40}$ Carbonate systems have attracted researchers' attention because of their biodegradability, biocompatibility, pH-sensitive dissolution along with easy-loading and functionalization. Their distinct advantages make them suitable as an antacid or drug delivery system in acidic conditions. Guix et al. ${ }^{41}$ had prepared biodegradable Janus $\mathrm{CaCO}_{3}$ MNMs which moved in acidic environments attributed to cancer cell growth. $\mathrm{CaCO}_{3}$ MNMs exhibited movement and dissolution in acidic conditions which were produced by quickly propagating HeLa cells. Baylis et al. ${ }^{42}$ proposed the first report of active self-fueled MNMs consisting of carbonate and tranexamic acid being used to transport drugs upstream through blood and deliver the functional protein cargo millimeters into the vasculature of wounds (as shown in Fig. 3C). The prepared MNMs generated vigorous reaction, and rapidly travelled through the whole blood through a combination of lateral propulsion, buoyant rise and convection, thus transporting drugs into the vasculature of wounds through flowing blood. The reaction was self-buffering because much of organic acid protonated tranexamic acid was neutralized as $\mathrm{CaCO}_{3}$ releasing $\mathrm{CO}_{2}$. MNMs with both decreasing blood loss and blood clotting functions were prepared by adsorbing thrombin onto porous $\mathrm{CaCO}_{3}$.

Choi et $a l .{ }^{43}$ had fabricated a biodegradable nanomotor for active drug delivery to cancer cells using a Pt deposited complex of $\mathrm{CaCO}_{3}$ and $\mathrm{CB}[6]$ conjugated hyaluronate ( $\mathrm{Pt} / \mathrm{CaCO}_{3} @$ HA-CB[6]). The biodegradable nanomotor was propelled by oxygen bubbles produced from Pt-catalyzed decomposition of $\mathrm{H}_{2} \mathrm{O}_{2} \cdot \mathrm{CaCO}_{3}$ as the main part of the drug delivery system was decomposed in tumor acidic microenvironments and the loaded HA-CB[6] was released. The drug loaded HA conjugate was taken into cancer cells through HA receptor-mediated endocytosis. The model drug was delivered to cancer cells via dual stimuli-responsive nanomotors: $\mathrm{pH}$-responsive dissolution of $\mathrm{CaCO}_{3}$ in tumor acidic microenvironments and cancer targeting capability of drug loaded HA-CB through HA receptors on cancer cells.

\section{Biocompatibility}

The biocompatibility of MNMs has attracted tremendous interest because biomedical MNMs are directly in contact with the body fluid, cells or tissues in the internal environment of the body. Biocompatibility is a term used to describe the ability of a material to perform in a specific application with an appropriate host response. $^{44}$ It is applicable for some nondegradable inorganic materials such as $\mathrm{Ti}, \mathrm{SiO}_{2}$, etc., and it is also applicable for lots of biodegradable materials. The typical examples of biodegradable and biocompatible MNMs are summarized in Table 1.

\subsection{Inorganic materials}

Besides the materials with both biodegradability and biocompatibility mentioned in Section 2, some representative nondegradable materials such as $\mathrm{Au}, \mathrm{Pt}, \mathrm{Fe}_{3} \mathrm{O}_{4}, \mathrm{SiO}_{2}$, graphene and hydroxyapatite have been proved to be biocompatible with organisms. The biocompatibility of these materials has made them good candidates for fabrication of MNMs for biomedical applications. As shown in Fig. 4A, Esteban-Fernández de Ávila and co-workers ${ }^{45}$ described ultrasound powered gold nanowires (Au NWs) loaded with an interfering RNA for gene silencing. DNA structures were wrapped on Au NWs and served as an anchor for siRNA. Once inside the cell, the loaded siRNA was responsible for rapidly suppressing target gene expression. Different from traditional siRNA carrier methods, these Au NW nanomotors capable of penetrating and traveling through the cell resulted in a 13 fold higher silencing response. A diversity of methods to prepare biocompatible MNMs including Janus micro-/nanostructures based on Au have been developed. For instance, Luo et al. ${ }^{46}$ fabricated self-propelled MNMs which consisted of multilayered assembly of ureases being asymmetrically immobilized on Janus Au/magnetic microparticles. The as-prepared MNMs could move much faster (5-fold) than monolayered motors. Besides, due to the intrinsic nature of magnetic microparticles, 
Table 1 Typical examples of biodegradable and biocompatible MNMs

\begin{tabular}{|c|c|c|c|c|c|c|}
\hline Main composition & Fabrication method & Design & $\begin{array}{l}\text { Biodegradation } \\
\text { method }\end{array}$ & $\begin{array}{l}\text { Propulsion } \\
\text { mechanism }\end{array}$ & Applications & Ref. \\
\hline \multicolumn{7}{|l|}{ Natural polymers } \\
\hline Alginate/chitosan & $\begin{array}{l}\text { Rolled-up technique and } \\
\text { template electrosynthesis }\end{array}$ & Nanorocket & - & $\mathrm{Pt}\left(\mathrm{H}_{2} \mathrm{O}_{2}\right)$ & Drug delivery & 10 \\
\hline Alginate/chitosan & $\begin{array}{l}\text { Template-assisted } \\
\text { layer-by-layer self-assembly }\end{array}$ & $\begin{array}{l}\text { Janus } \\
\text { microcapsule }\end{array}$ & - & Light (NIR, Au) & $\begin{array}{l}\text { Potential biomedical } \\
\text { application }\end{array}$ & 11 \\
\hline $\begin{array}{l}\text { Poly(vinyl alcohol)/alginate } \\
\text { (main component) }\end{array}$ & Gelation & $\begin{array}{l}\text { Star-shaped } \\
\text { hydrogel } \\
\text { micromotor }\end{array}$ & Amylase & $\begin{array}{l}\text { Magnetic field } \\
\left(\mathrm{Fe}_{3} \mathrm{O}_{4}\right)\end{array}$ & Drug delivery & 12 \\
\hline $\begin{array}{l}\text { Bovine serum albumin/ } \\
\text { poly-L-lysine }\end{array}$ & $\begin{array}{l}\text { Template-assisted } \\
\text { layer-by-layer self-assembly }\end{array}$ & Rocket & $\alpha$-Chymotrypsin & $\begin{array}{l}\text { Light }(\mathrm{NIR}, \mathrm{Au}) \\
\text { propulsion/ } \\
\text { magnetic guidance }\end{array}$ & Drug delivery & 13 \\
\hline $\begin{array}{l}\text { Human serum albumin/ } \\
\text { urease }\end{array}$ & $\begin{array}{l}\text { Template-assisted } \\
\text { layer-by-layer self-assembly }\end{array}$ & Microtube & Proteases & Enzyme (urease) & $\begin{array}{l}\text { Potential biomedical } \\
\text { application }\end{array}$ & 14 \\
\hline Squid ring teeth proteins & $\begin{array}{l}\text { Extraction and purification, } \\
\text { preparation of motor } \\
\text { solution, protein film } \\
\text { casting and laser } \\
\text { micromachining }\end{array}$ & $\begin{array}{l}\text { Natural structure } \\
\text { of Squid ring } \\
\text { teeth proteins }\end{array}$ & $\begin{array}{l}\mathrm{pH} \text { stimulus and } \\
\text { proteolysis }\end{array}$ & $\begin{array}{l}\text { Marangoni } \\
\text { propulsion/ } \\
\text { magnetic steer (iron } \\
\text { oxide nanoparticles) }\end{array}$ & $\begin{array}{l}\text { Environmental } \\
\text { remediation and } \\
\text { drug delivery }\end{array}$ & 15 \\
\hline \multicolumn{7}{|l|}{ Synthetic polymers } \\
\hline Methacryloyl & $3 \mathrm{D}$ print & $\begin{array}{l}\text { Soft helical } \\
\text { micromotor }\end{array}$ & Collagenase & $\begin{array}{l}\text { Magnetic field } \\
\left(\mathrm{Fe}_{3} \mathrm{O}_{4}\right)\end{array}$ & $\begin{array}{l}\text { Support cell } \\
\text { attachment and } \\
\text { growth }\end{array}$ & 19 \\
\hline Polycaprolactone & Self-assembly & $\begin{array}{l}\text { Polymer single } \\
\text { crystals }\end{array}$ & Lipase & $\mathrm{Ag}\left(\mathrm{H}_{2} \mathrm{O}_{2}\right)$ & $\begin{array}{l}\text { Detection of a } \\
\text { solute, drug delivery }\end{array}$ & 20 \\
\hline Polycaprolactone/catalase & Self-seeding & $\begin{array}{l}\text { Polymer single } \\
\text { crystals }\end{array}$ & Lipase & Catalase $\left(\mathrm{H}_{2} \mathrm{O}_{2}\right)$ & Gas sensing & 21 \\
\hline $\begin{array}{l}\text { Polycaprolactone/sodium } \\
\text { 1-dodecanesulfonate }\end{array}$ & One-step electrospinning & $\begin{array}{l}\text { Spindle-like } \\
\text { micromotor }\end{array}$ & Lipase & Marangoni effect & pH sensing & 22 \\
\hline Polycaprolactone & $\begin{array}{l}\text { Single emulsion and } \\
\text { solvent evaporation } \\
\text { method }\end{array}$ & Motherships & Lipase & $\mathrm{Pt}\left(\mathrm{H}_{2} \mathrm{O}_{2}\right)$ & $\begin{array}{l}\text { Targeted cargo- } \\
\text { carrying nanospheres } \\
\text { delivery }\end{array}$ & 23 \\
\hline $\begin{array}{l}\text { Poly(ethylene glycol)- } b \text { - } \\
\text { poly( } \varepsilon \text {-caprolactone)/ } \\
\text { poly(ethylene glycol)- } b \text { - } \\
\text { polystyrene }\end{array}$ & Self-assembly & Stomatocyte & pH stimulus & $\mathrm{Pt}\left(\mathrm{H}_{2} \mathrm{O}_{2}\right)$ & Drug delivery & 24 \\
\hline $\begin{array}{l}\text { Poly(ethylene glycol)- } b \text { - } \\
\text { poly(D,L-lactide) }\end{array}$ & Self-assembly & Stomatocyte & - & $\begin{array}{l}\text { Glucose oxidase } \\
\text { (glucose)/catalase } \\
\left(\mathrm{H}_{2} \mathrm{O}_{2}\right)\end{array}$ & - & 25 \\
\hline Poly(lactic-co-glycolic acid) & Electrospray technique & Microparticle & - & Catalase $\left(\mathrm{H}_{2} \mathrm{O}_{2}\right)$ & Drug delivery & 26 \\
\hline $\begin{array}{l}\text { Poly(lactic-co-glycolic acid)/ } \\
\text { polyethylene oxide }\end{array}$ & $\begin{array}{l}\text { Coelectrospinning and } \\
\text { cryosectioning }\end{array}$ & Rocket & - & $\begin{array}{l}\mathrm{Pt} / \mathrm{Ag} / \text { catalase } \\
\left(\mathrm{H}_{2} \mathrm{O}_{2}\right)\end{array}$ & $\begin{array}{l}\text { Potential biomedical } \\
\text { application }\end{array}$ & 27 \\
\hline $\begin{array}{l}\text { Poly(ethylene glycol)- } b \text { - } \\
\text { poly(D,L-lactide) }\end{array}$ & Self-assembly & Nanotube & - & Catalase $\left(\mathrm{H}_{2} \mathrm{O}_{2}\right)$ & Drug delivery & 28 \\
\hline \multicolumn{7}{|l|}{ Biodegradable metals } \\
\hline $\mathrm{Al}-\mathrm{Ga}$ & $\begin{array}{l}\text { Template-assisted } \\
\text { electrodeposition }\end{array}$ & $\begin{array}{l}\text { Janus } \\
\text { micromotor }\end{array}$ & Reaction with water & $\mathrm{Al}\left(\mathrm{H}_{2} \mathrm{O}\right)$ & $\begin{array}{l}\text { Potential biomedical } \\
\text { application }\end{array}$ & 32 \\
\hline $\mathrm{Fe} / \mathrm{Zn}$ & $\begin{array}{l}\text { Template-assisted } \\
\text { electrodeposition }\end{array}$ & Microtube & $\begin{array}{l}\text { Reaction with gastric } \\
\text { acid }\end{array}$ & $\mathrm{Zn}\left(\mathrm{H}^{+}\right)$ & Drug delivery & 33 \\
\hline Poly(aspartic acid)/Fe/Zn & $\begin{array}{l}\text { Template-assisted } \\
\text { electrodeposition and } \\
\text { electrostatic interaction }\end{array}$ & Rocket & $\begin{array}{l}\text { Reaction with gastric } \\
\text { acid/proteases }\end{array}$ & $\mathrm{Zn}\left(\mathrm{H}^{+}\right)$ & Drug delivery & 34 \\
\hline $\mathrm{Mg} / \mathrm{ZnO}, \mathrm{Mg} / \mathrm{Si}, \mathrm{Zn} / \mathrm{Fe}, \mathrm{Zn}$ & $\begin{array}{l}\text { Template-assisted } \\
\text { electrodeposition }\end{array}$ & $\begin{array}{l}\text { Janus } \\
\text { micromotor }\end{array}$ & $\begin{array}{l}\text { Reaction with gastric } \\
\text { acid/water }\end{array}$ & $\begin{array}{l}\mathrm{Mg}\left(\mathrm{H}_{2} \mathrm{O}\right), \mathrm{Zn}\left(\mathrm{H}^{+}\right), \\
\mathrm{Fe}\left(\mathrm{H}^{+}\right)\end{array}$ & $\begin{array}{l}\text { Potential biomedical } \\
\text { application }\end{array}$ & 36 \\
\hline Enteric polymer/Mg & $\begin{array}{l}\text { Template-assisted } \\
\text { electrodeposition }\end{array}$ & Microtube & $\begin{array}{l}\text { Reaction with } \\
\text { intestinal fluids }\end{array}$ & $\mathrm{Mg}\left(\mathrm{H}_{2} \mathrm{O}\right)$ & Drug delivery & 37 \\
\hline $\begin{array}{l}\text { Poly(lactic-co-glycolic acid)/ } \\
\text { alginate/chitosan/Mg } \\
\text { Carbonate systems }\end{array}$ & $\begin{array}{l}\text { Template-assisted } \\
\text { layer-by-layer self-assembly }\end{array}$ & $\begin{array}{l}\text { Janus } \\
\text { micromotor }\end{array}$ & Reaction with water & $\mathrm{Mg}\left(\mathrm{H}_{2} \mathrm{O}\right)$ & Antibacterial activity & 38 \\
\hline $\mathrm{CaCO}_{3}$ & $\begin{array}{l}\text { Inorganic chemical } \\
\text { reaction }\end{array}$ & $\begin{array}{l}\text { Janus } \\
\text { micromotor }\end{array}$ & $\begin{array}{l}\text { Reaction in the acidic } \\
\text { microenvironment } \\
\text { generated by cancer } \\
\text { cells }\end{array}$ & $\mathrm{CaCO}_{3}\left(\mathrm{H}^{+}\right)$ & $\begin{array}{l}\text { Potential biomedical } \\
\text { application }\end{array}$ & 41 \\
\hline $\mathrm{CaCO}_{3}$ & $\begin{array}{l}\text { Inorganic chemical } \\
\text { reaction }\end{array}$ & Particle & $\begin{array}{l}\text { Reaction with loaded } \\
\text { drug tranexamic acid }\end{array}$ & $\begin{array}{l}\mathrm{CaCO}_{3} \text { (tranexamic } \\
\text { acid) }\end{array}$ & Drug delivery & 42 \\
\hline $\mathrm{CaCO}_{3} /$ hyaluronate & Chemical reaction & $\begin{array}{l}\text { Janus } \\
\text { nanoparticles }\end{array}$ & $\begin{array}{l}\text { Reaction in the acidic } \\
\text { microenvironment } \\
\text { generated by cancer } \\
\text { cells }\end{array}$ & $\mathrm{Pt}\left(\mathrm{H}_{2} \mathrm{O}_{2}\right)$ & Drug delivery & 43 \\
\hline $\mathrm{Au}$ & $\begin{array}{l}\text { Template-assisted } \\
\text { electrodeposition }\end{array}$ & Nanowires & - & $\begin{array}{l}\text { Ultrasound } \\
\text { propulsion }\end{array}$ & Gene silencing & 45 \\
\hline
\end{tabular}


Table 1 (continued)

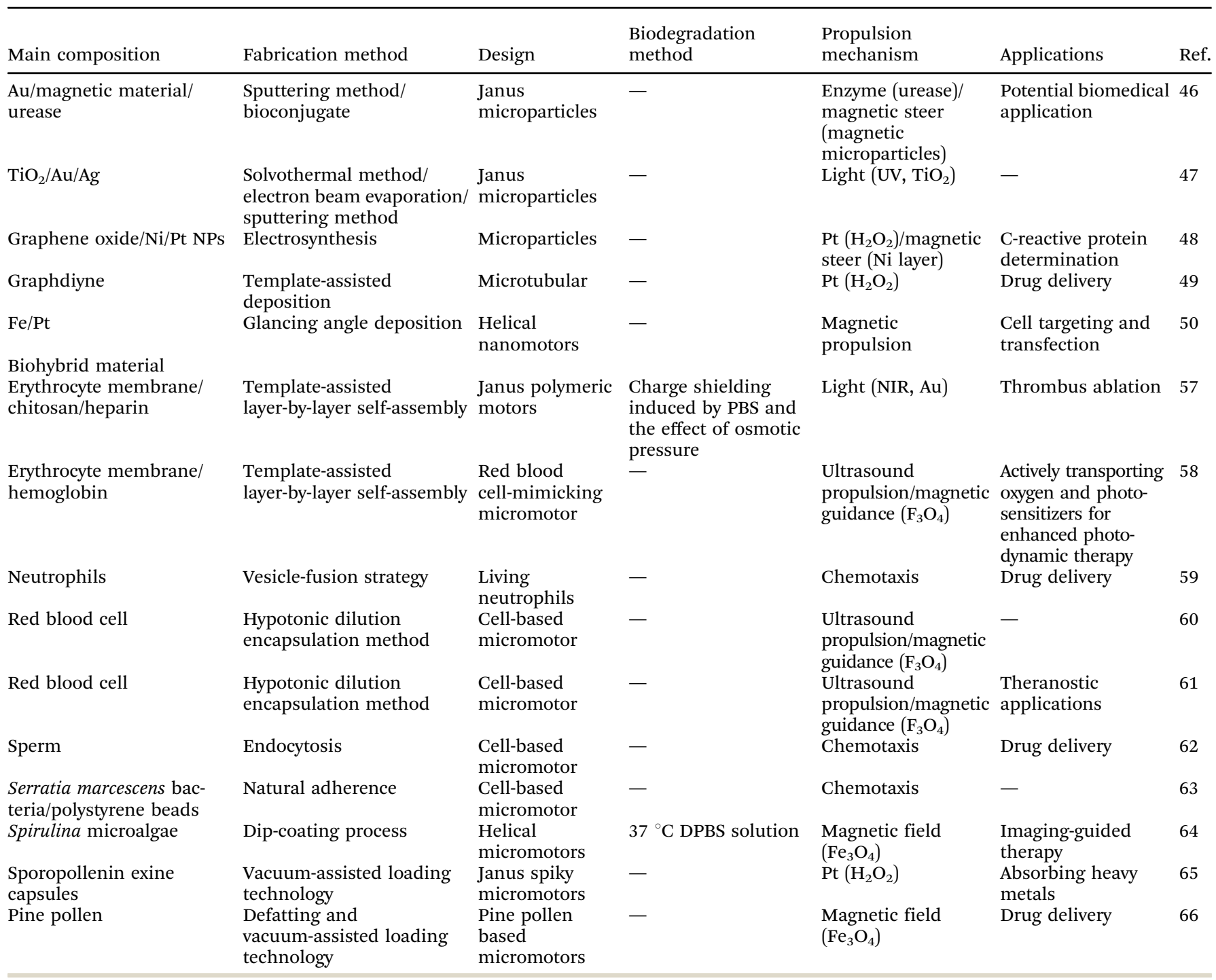

the as-obtained MNMs exhibited controllable motion under an external magnetic field. $\mathrm{Au} / \mathrm{Ag}$ bimetallic coating was also employed to enhance the speed of $\mathrm{TiO}_{2}$ Janus MNMs. ${ }^{47}$ The two metal layers ( $\mathrm{Au} / \mathrm{Ag}$ bimetallic coating) worked in photocatalytic synergy to increase the speed of as-prepared MNMs 2-fold compared to either metal alone. Molinero-Fernández and co-workers ${ }^{48}$ have proposed MNM-based immunoassay for C-reactive protein determination. The MNMs with analysis ability contained three layers, outer layer (reduced graphene oxide for antibody functionalization), interlayer (Ni for magnetic control) and inner layer (Pt NPs for catalytic decomposition of $\mathrm{H}_{2} \mathrm{O}_{2}$ for bubble propulsion). Reduced graphene oxide allowed immobilizing biomolecules on the surface and high MNM propulsion efficiency when catalysts used in the inner layer. Although graphene and graphene oxide as the widely studied 2D nanomaterials had been widely used for biomedical applications, some graphene analogs such as graphdiyne exhibited improved biocompatibility and lower toxicity than graphene. Yuan et l. $^{49}$ illustrated the use of graphdiyne based MNMs for drug delivery and sensing of toxins. The tubular structures of graphdiyne based
MNMs were fabricated by template deposition on membrane templates (as indicated in Fig. 4B). The inner Pt patch was used for propulsion at $\mathrm{H}_{2} \mathrm{O}_{2}$ solutions. In the case of moving MNMs, high cell viability (almost 100\%) was noted when incubated with HeLa cells. The traditional magnetic materials such as $\mathrm{Ni}$ and $\mathrm{Fe}_{3} \mathrm{O}_{4}$ suffer from some drawbacks due to either toxic nature or weak magnetization. Kadiri et $a l^{50}$ demonstrated ferromagnetic helical MNMs which were prepared by co-depositing $\mathrm{Fe}$ and $\mathrm{Pt}$ onto $\mathrm{SiO}_{2}$ in one single annealing procedure. As shown in Fig. 4C, the obtained nanopropellers containing ferromagnetic $\mathrm{L}_{0}$ FePt could be magnetically steered to a specific cell and achieved cell transfection. Cancer cells which were incubated with helices expressed enhanced green fluorescent protein than control cells. Besides, nanopropellers have been proved to be noncytotoxic and fully biocompatible with A549 and HEK cells.

\subsection{Biohybrid material}

The practical use of MNMs in organisms requires the development of fully biocompatible designs facilitating MNMs' movement in 

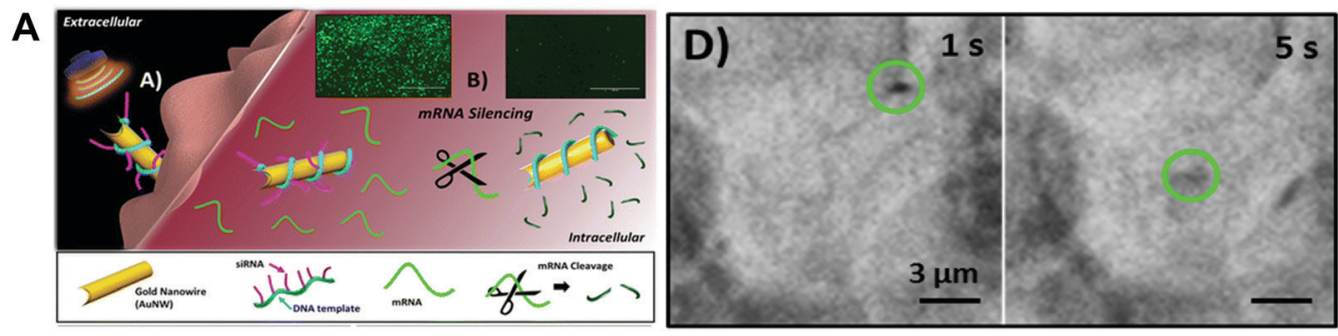

B
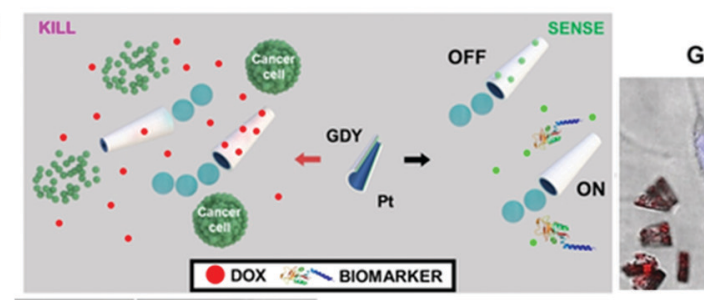

GDY-Pt

Static DOX-

Moving DOX-

GDY-Pt

GDY-Pt

C
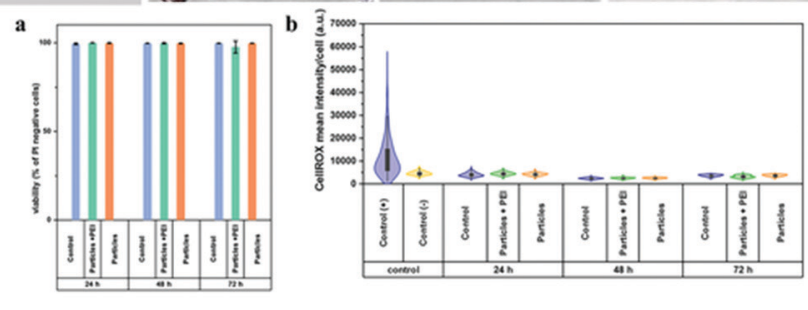

Fig. 4 Inorganic material based biocompatible MNMs. (A) Schematic of the nanomotor-based gene silencing approach and optical images of the acoustic movement of the motors inside living cells. (B) Schematic of the graphdiyne micromotors for DOX delivery, cancer cell killing, fluorescent "OFFON" sensing of toxins, confocal fluorescence microscopy images of motors' in vitro cancer cell killing ability and control experiments. (C) Illustration of FePt nanopropellers, cytotoxicity and oxidative stress of these propellers. (A) Reproduced with permission. ${ }^{45}$ Copyright 2016, American Chemical Society. (B) Reproduced with permission. ${ }^{49}$ Copyright 2020, Wiley-VCH. (C) Reproduced with permission. ${ }^{50}$ Copyright 2020, Wiley-VCH.

the internal environment without producing harmful effects and achieving the desired functions at the target location. The application of biohybrid materials to synthetic MNMs has resulted in biomimetic micromotors with enhanced capabilities and functionalities. The different designs of biohybrid MNMs in recent years are divided into three groups: cell membranecoated MNMs, cell-based MNMs and plant-based MNMs. ${ }^{51,52}$ Due to the inherent biocompatibility of biohybrid materials, the fabricated biohybrid MNMs cannot trigger an immune reaction while taking advantages of the robust but random autonomous movement of synthetic components or depending on slow but chemotactic movement of biological components. The coupling of biohybrid materials and synthetic components has led to active biohybrid MNMs with expanded therapeutic and detoxification capabilities compared with individual biological or artificial components.

The application of membranes derived from natural cells to the synthetic micro/nanoparticles allows researchers to easily achieve native biological functions. $\mathrm{Hu}$ et al..$^{53}$ reported a biomimetic approach for nanoparticle functionalization by extruding biodegradable PLGA nanoparticles in the presence of erythrocyte membranes. The prepared erythrocyte-mimicking nanoparticles with the erythrocyte exterior showed superior circulation halflife while retaining the applicability of the PLGA core. The successful translocation of erythrocyte membranes and their functionalities to the surface of nanoparticles lay the foundation for future study. Hu et al. ${ }^{54}$ also presented biomimetic toxin nanosponges with a PLGA core wrapped in natural erythrocyte membranes. The proposed toxin nanosponges functioned as a toxin decoy in vivo. The natural erythrocyte membranes acted as a substrate mimicry to absorb pore-forming toxins (staphylococcal alpha-haemolysin) and diverted the toxins away from their cellular targets. The inner PLGA core in nanosponges stabilized the erythrocyte membrane shell, so as to realize the prolonged systemic circulation and absorb membrane-damaging toxins in organisms. This nanotoxoid platform was generalized for the neutralization and delivery of non-disrupted pore-forming toxins to create safe and effective vaccination. Compared with vaccination with toxin neutralization by heat, the proposed nanotoxoid vaccine exhibited stronger immunogenicity and superior efficacy. ${ }^{55}$ Chen et $a l .{ }^{56}$ tested the erythrocyte membrane-coated nanosponges with different pore-forming toxins including melittin and found that they were effective in completely neutralizing toxins' hemolytic activity. Furthermore, the nanosponge-detained toxins posed negligible toxicity to cells and live animals.

Recently, the proposed cell membrane coating technology has been utilized in MNMs for numerous medical applications. Shao et al. ${ }^{57}$ fabricated erythrocyte membrane-coated MNMs which were triggered by NIR laser irradiation. CHI and heparin were used as naturally occurring building blocks to construct capsules via LbL assembly technology. In addition, heparin was used not only as a building block but also as a therapeutic agent for thrombus ablation. NIR laser propulsion was achieved by partially coating the capsules with a gold shell. The reversible 
"on/off" motion of MNMs and their extent of movement were tuned by NIR laser intensity. Again, NIR irradiation can also be used for photothermal ablation of thrombus. To avoid immune response caused by the foreign materials, MNMs were coated with the erythrocyte membrane. Through coating with the erythrocyte membrane, MNMs exhibited a long-circulation behavior in a biological environment. Due to the combined properties of the fabricated MNMs, these biocompatible motors exhibited good performance in thrombus ablation in vivo. Gao et $a .^{58}$ reported red blood cell-mimicking MNMs with oxygen- and photosensitizer-carrying capacity for enhancing photodynamic therapy. The red blood cell-mimicking MNMs consisted of red blood cell-shaped hemoglobin, $\mathrm{Fe}_{3} \mathrm{O}_{4}$ nanoparticles, indocyanine green and the red blood cell membrane. These MNMs were navigated by an external magnetic field and propelled by ultrasound. The prepared MNMs were capable of avoiding biofouling and immune clearance, actively transporting oxygen and photosensitizers for photodynamic therapy.

In addition to red blood cells, other blood cells can also be transformed into biohybrid MNMs with excellent biocompatibility. For instance, living neutrophils with intrinsic chemotaxis ability had been transformed into autonomous biohybrid MNMs for targeted drug transport. Shao et $a .^{59}$ had cloaked drug-loaded mesoporous silica nanoparticles with $E$. coli membranes and then incubated them with neutrophils to prepare biohybrid neutrophil MNMs via an engulfing process (as shown in Fig. 5A). Camouflaging of mesoporous silica with $E$. coli membranes not only reduced the leakage of the model drug from nanoparticles, but also enabled the uptake of drug-loaded nanoparticles into neutrophils without loss of cell viability as well as chemotaxis capability. The fabricated biohybrid MNMs exhibited chemotaxis toward chemoattractant gradients caused by $E$. coli.
Cell-based MNMs are expected to bridge the gap between artificial MNMs and biological world. As indicated in Fig. 5B, Wu et al. ${ }^{60}$ presented a natural red blood cell-based motor with ultrasound powered and magnetically guided propulsion. The proposed motors were fabricated via the hypotonic dilution encapsulation method to load magnetic nanoparticles into red blood cells. Due to the asymmetric distribution of magnetic nanoparticles within the cells, a net magnetization was produced and allowed magnetic alignment and guidance under ultrasound propulsion. The prepared red blood cell-based motor exhibited controlled motion through whole blood and avoided the detection and uptake by macrophages. Then on the basis of this research, Wu et al. ${ }^{61}$ proposed a new red blood cell-based motor capable of transporting theranostic cargoes. Quantum dots, DOX and magnetic nanoparticles were simultaneously encapsulated within red blood cells. The magnetically guided acoustic propelled transport of multicargo-loaded red blood cells was demonstrated via a microfluidic channel.

The chemotactic movement of sperm cells was implemented to develop self-propelled MNMs for application in biomedical fields. The natural sperm cells were translated into MNMs by functionalizing them with different synthetic payloads, such as CdSe/ZnS quantum dots, fluorescein isothiocyanate modified Pt NPs and DOX coated iron-oxide nanoparticles, through a well-known endocytosis process. ${ }^{62}$ The sperm cell-based MNMs acted as a power source for movement under the guidance of egg secretions. The flagellar lengths of the sperm motors were tailored by adjusting the solution osmolarity that would lead to changes in the speed of motors. The drug loading and releasing ability of motors was studied in an acidic environment where a change in cell membrane permeability of sperm allowed the release of loaded drug. The potential biomedical utility of

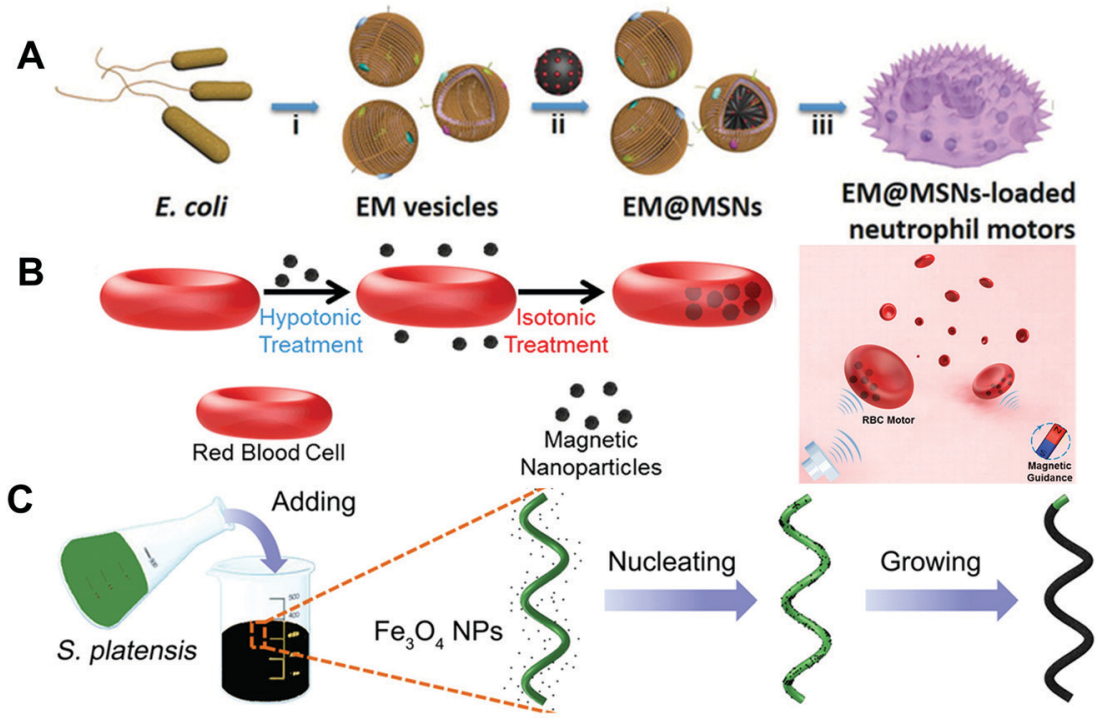

Fig. 5 Biohybrid material based MNMs. (A) Schematic of the synthesis process of neutrophil micromotors composed of mesoporous silica nanoparticles coated with E. coli membranes. (B) Schematic illustration of preparation of red blood cell-based micromotors in whole blood. (C) Schematic of the dip-coating process of $\mathrm{S}$. platensis in a suspension of $\mathrm{Fe}_{3} \mathrm{O}_{4} \mathrm{NPs}$ and time-lapse image sequences of controlled locomotion. (A) Reproduced with permission. ${ }^{59}$ Copyright 2017, Wiley-VCH. (B) Reproduced with permission. ${ }^{60}$ Copyright 2014, American Chemical Society. (C) Reproduced with permission. ${ }^{64}$ Copyright 2017, The Authors, some rights reserved; exclusive licensee American Association for the Advancement of Science. 
sperm motors was illustrated using SKOV-3 ovarian cancer cells. Zhuang et $a l .{ }^{63}$ developed the multi-bacteria-driven MNMs, studied their chemotaxis behavior towards the chemoattractant L-serine and elucidated their associated collective chemotaxis behavior through statistical analysis. A heading bias was found on the proposed MNMs under the L-serine gradient and it was the crucial reason that led to the cooperative chemotaxis behavior among the attached bacteria. The chemotactic drift velocity of motors increased super quadratically with their mean speed.

Other biological substances such as plant tissues are also involved as constituents of MNMs. Yan et al. ${ }^{64}$ reported a type of MNMs endowed with multifunctional capabilities by dip-coating of Spirulina microalgae in magnetite suspensions (as shown in Fig. 5C). The multifunctional capabilities of MNMs aimed at imaging-guided therapy including in vivo imaging capability (fluorescence imaging and inherent magnetic resonance contrast), biodegradation (degradation time span was adjusted by controlling dip-coating time) and selective cytotoxicity to cancer cells. Furthermore, a swarm of MNMs inside the rat's stomach were propelled by an external magnetic field and could be observed via magnetic resonance imaging. Wang et al. ${ }^{65}$ proposed the plant-based MNMs with unique spiky morphology. The spiky MNMs consisted of a BSA-loaded sporopollenin exine capsules' body and were partially coated with Pt on their surface. These bioinspired MNMs were powered by oxygen bubbles produced by Pt-catalyzed decomposition of $\mathrm{H}_{2} \mathrm{O}_{2}$. Furthermore, the bioinspired MNMs with a large internal cavity could serve as natural capsules for macromolecular encapsulation via suction through the pores using vacuum-assisted loading technology. Moreover, sporopollenin with a polymeric porous structure was capable of absorbing heavy metals from water. Sun $e t a l^{66}$ reported pine pollen-based MNMs and studied their potential applications in biomedical fields. The proposed biohybrid MNMs were fabricated by encapsulating both magnetic nanoparticles and drugs within two hollow air sacs of pine pollen using vacuum-assisted loading technology. The loaded magnetic nanoparticles endowed the individual and swarm MNMs with precisely and controlled movement in complex biological fluids through regulating the external magnetic field. In addition, the biohybrid MNMs were triggered to release the loaded drug on demand. Magnetic nanoparticles aggregated into rods in a high-intensity magnetic field, which would generate a fluid field to release the loaded drug when a rotating magnetic field was applied.

The biohybrid MNMs based on synthetic or biological components have been developed for diverse applications. The biohybrid materials themselves (cell membranes, cells or plant tissues) have absolute advantages in biocompatibility and bioactivity due to their biological nature, which can endow the MNMs with functionalities such as versatile drug delivery, improved and prolonged propulsion in complex biofluids and an accelerated biodetoxification process.

\section{Biosafe propulsion}

The remarkable performance of MNMs with various propulsion mechanisms has provided an inspiration for development of artificial MNMs to achieve different applications. For the biomedical application, it is quite essential to propel MNMs in a variety of complex biofluids. The characteristics of locally supplied fuels, chemical reactions and external fields that offer propulsion energy to fuel or fuel-free MNMs should be biosafe, ease of application and feasible within the organisms. Two main MNMs based on propulsion mechanisms, such as biosafe fuel-powered MNMs and fuel-free MNMs (external field actuation and biohybrid MNMs), are discussed in this section.

\subsection{Biosafe fuel-powered MNMs}

A variety of MNMs are based on biosafe fuel powered motors. There exist two means, asymmetric chemical gradient and bubble thrust, to achieve biosafe fuel propulsion of MNMs. The general design strategy for biosafe fuel-powered MNMs is to establish an asymmetric structure to form a chemical gradient and bubble thrust, and select suitable chemical reactions with a rapid product formation rate to power MNMs. ${ }^{67,68}$

Chemically powered MNMs based on catalytic reactions result in an asymmetric chemical gradient (concentration, electrical or temperature) and the gradient induces the movement. As indicated in Fig. 6A, Sugai et al. ${ }^{14}$ presented the "all protein" microtubes comprising human serum albumin and Ure enzyme. These biodegradable microtube motors could be fully digested by proteases. Their driving force was the concentration gradient of the products $\left(\mathrm{NH}_{2} \mathrm{CONH}_{2}+\mathrm{H}_{2} \mathrm{O} \rightarrow 2 \mathrm{NH}_{3}+\mathrm{CO}_{2}\right)$ from onedimensional pore space to the bulk aqueous solution. Bubble thrust is a very important aspect in biosafe fuel powered MNMs. Bubble propelled MNMs are usually based on noble metal-enabled catalysis and chemical reactions between active metals and water. Their movement relies on decomposition of $\mathrm{H}_{2} \mathrm{O}_{2}{ }^{69}$ water, ${ }^{32}$ urea $^{70}{ }^{7}$ gastrointestinal fluid ${ }^{71}$ and other emerging materials ${ }^{72}$ to generate bubbles. In addition, for applying $\mathrm{H}_{2} \mathrm{O}_{2}$ chemical fuel in realistic physiological conditions, excessive $\mathrm{H}_{2} \mathrm{O}_{2}$ may induce oxidative stress and damage of living tissues. Therefore, alternative powered methods to reduce the toxicity of $\mathrm{H}_{2} \mathrm{O}_{2}$ fuel or development of other biocompatible fuels is desired. As indicated in Fig. $6 \mathrm{~B}, \mathrm{Wu}$ and co-workers ${ }^{69}$ presented a kind of catalase functionalized Janus MNM which could be self-propelled through biocatalytic decomposition of $\mathrm{H}_{2} \mathrm{O}_{2}$ at low concentration $(0.1 \%$, a biocompatible concentration). A microtube motor consisting of poly(aspartic acid), an Fe intermediate layer and a core of Zn was fabricated by Zhou et $a l^{34}$ The proposed microrocket could be actuated using gastric acid as fuel, and be magnetically guided to induce prospective tracks and for targeted and sustained release of the loaded drug in the acidic stomach environment. After fulfilling the tasks, the microtube was completely degraded by gastric acid or proteases in the digestive tract. Wan et al..$^{72}$ had proposed NO-propelled MNMs in which the reactant and by-products all provided beneficial effects on the human body (as shown in Fig. 6C). The prepared zero-waste and selfdestroyed MNMs utilized L-arginine as fuel for the conversion of NO. In addition, the framework of the NO-propelled MNMs including hyperbranched polyamide also served as a nontoxic fluorescent dye to tract the vehicle in vitro.

Biosafe fuel propulsion is well developed for MNMs. However, it should be noted that MNMs propelled by chemical 

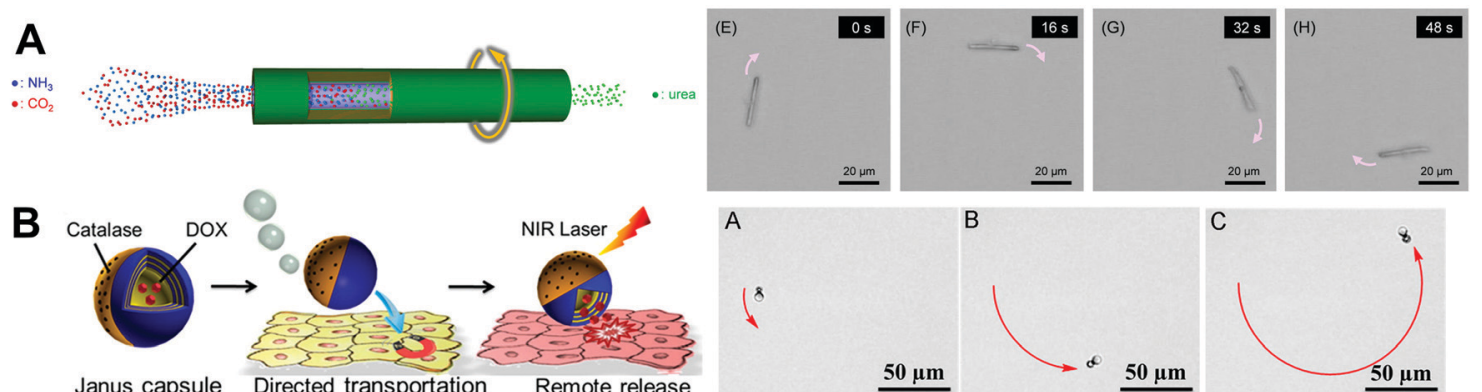

C

Janus capsule
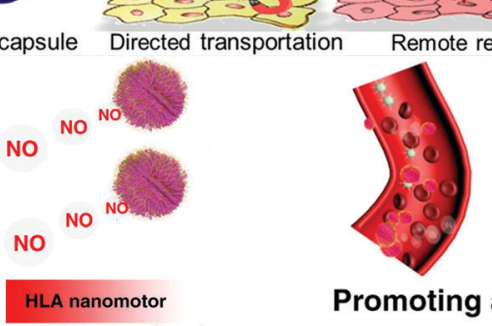

Promoting angiogenesis

L-citrulline

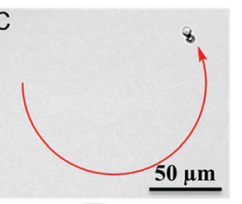

$\underline{\mathbf{5 0} \mu \mathrm{m}}$

NO

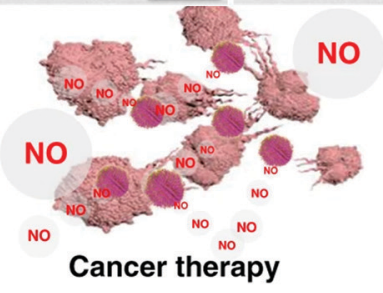

Fig. 6 Biosafe fuel-powered MNMs. (A) Schematic illustration of a swimming Avi/bUre microtube with nonbubble propulsion and self-rotation, and micrographs of microscopic observations of self-propelled microtubes. (B) Scheme of the targeted movement and light-triggered drug release of Janus capsule motors, and time-lapse images of self-propulsion of Janus motors. (C) Schematic illustration of the formation of a zwitterion-based nanomotor and the NO generation principle. (A) Reproduced with permission. ${ }^{14}$ Copyright 2019, Wiley-VCH. (B) Reproduced with permission. ${ }^{69}$ Copyright 2014 , American Chemical Society. (C) Reproduced with permission. ${ }^{72}$ Copyright 2019, Springer Nature.

reaction between MNMs and chemical fuels would make the movement difficult to be reversible and controllable. In the bubble propulsion model, their applicability suffers drawbacks such as the possible gas embolism induced by the generated bubbles and the short lifetime caused by depletion of the propellant. $^{73}$ Therefore, it is essential to develop biosafe fuel powered MNMs using in vivo fuels and monitor their surrounding chemical environment.

\subsection{Fuel-free MNMs}

Fuel-free MNMs propelled by external fields and living organisms have shown good performance in achieving complex tasks due to their precise controlled movement, long lifetime and excellent biocompatibility compared with fuel powered MNMs. The external fields that provide energy to power MNMs can be classified according to their sources into light, ultrasound and magnetic fields. MNMs propelled by living organisms have attracted much attention because of their excellent biocompatibility and self-propulsion.

Light-powered MNMs. The application of light-driven MNMs has addressed some of the limitations in chemical propulsion, and light as a renewable and easily controlled energy source has been widely used to power MNMs with high spatial and temporal resolution. ${ }^{74}$ The mechanism of light powered MNMs is usually referred to as photothermal propulsion. Gold as a kind of noble metal is one of the most widely used light-responsive materials in MNMs' design. Light-triggered thermophoresis is employed to power MNMs using a light induced temperature gradient. As illustrated in Fig. 7A, Xuan et al. ${ }^{11}$ had reported a Janus micromotor powered by light. GNRs were modified on one side of $\mathrm{CHI} /$ ALG multilayer capsules to generate a thermal gradient due to their photothermal effect and thus propelled the micromotors under NIR illumination.
Light-powered MNMs have overcome some inherent limitations in chemical propulsion. However, light-powered MNMs are still accompanied by some drawbacks which should be considered. For example, the limited penetration of light in tissues may restrict their biomedical application.

Ultrasound-powered MNMs. Ultrasound has been widely used for basic and clinical research due to its noninvasive and harmless properties. The utility of ultrasound energy in MNM propulsion has provided a robust actuation strategy and fast motion even in complex biofluids. ${ }^{75}$ Gao et al..$^{58}$ fabricated an ultrasound propelled and magnetically guided red blood cellmimicking micromotor (as shown in Fig. 7B). The asymmetric structure and high material density resulted in pressure gradients and thus effectively affected the motion speed of prepared MNMs compared with normal red blood cells. Upon exposure to an ultrasound field, the obtained MNMs could move autonomously in biological media with a speed up to $56.5 \mu \mathrm{m} \mathrm{s}^{-1}$ (28.2 body lengths $\mathrm{s}^{-1}$ ) by converting ultrasound energy into mechanical force. However, ultrasound actuation suffers from specialized equipment, limited chamber size and difficulties in controlling the motion of individual MNMs.

Magnetically powered MNMs. Magnetic propulsion has been widely used in the biomedical field for powering MNMs because magnetic fields with low strength are harmless to cells and tissues. Magnetic fields can be used not only to control the directional motion but also to propel MNMs. The prevalent number of magnetically propelled MNMs are with helical or flexible structures. ${ }^{76-78}$ Wang et al. ${ }^{19}$ presented 3D printed biodegradable soft helical MNMs. The soft MNMs with helical geometry were decorated with magnetic nanoparticles and rendered magnetically responsive (as shown in Fig. 7C). Compared with rigid helical MNMs, the soft helical MNMs could get into the corkscrew region stably with a relatively high forward velocity as 


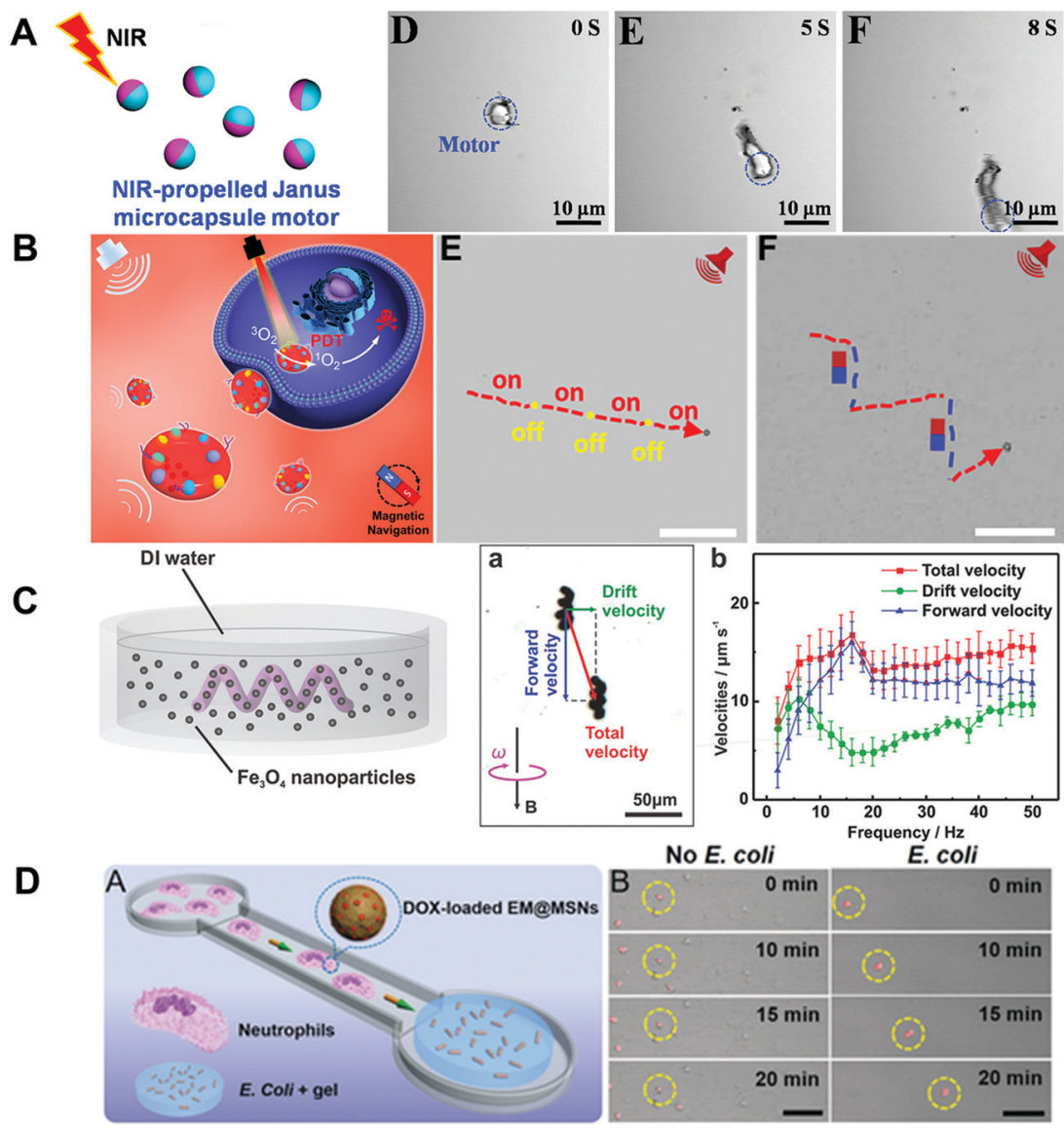

Fig. 7 Fuel-free MNMs. (A) Schematic illustration of Janus (CHI/ALG) 5 capsule micromotors and video frames of these micromotors under continuous NIR laser illumination. (B) Schematic illustration of a red blood cell-mimicking micromotor and "on/off" motion of micromotors in serum propelled by an external ultrasound field. (C) Schematic illustration of GelMA helical micromotors decorated with magnetic nanoparticles and swimming characterization of these micromotors. (D) Chemotactic motion of hybrid neutrophil micromotors and time-lapse CLSM images of these motors with and without $E$. coli. (A) Reproduced with permission. ${ }^{11}$ Copyright 2015, Elsevier B.V. (B) Reproduced with permission. ${ }^{58}$ Copyright 2019, American Chemical Society. (C) Reproduced with permission. ${ }^{19}$ Copyright 2018, Wiley-VCH. (D) Reproduced with permission. ${ }^{59}$ Copyright 2017, Wiley-VCH.

the rotating frequency of the magnetic field continuously increased, suggesting a unique self-adaptive swimming behavior. Magnetic propulsion also suffers from difficulties in the complex fabrication route and the demand for specialized equipment.

MNMs driven by living organisms. There has been considerable interest in the fabrication of biohybrid MNMs which combine organism with artificial micro/nanostructures. Biohybrid MNMs are capable of expanding the scope of movement of synthetic MNMs in complex biofluids, and may provide a better understanding of the natural movement mechanisms at the micro-/nanoscale. ${ }^{2}$ Biohybrid MNMs constitute an important alternative to synthetic MNMs due to their self-propulsion, excellent biocompatibility and biodegradability. Shao et al. ${ }^{59}$ presented biohybrid MNMs fabricated by integrating drugloaded mesoporous silica nanoparticles with neutrophils. The resulting hybrid MNMs showed chemotactic behavior induced by a gradient of chemoattractant secreted by $E$. coli. Besides, the activated neutrophils could also remove dead $E$. coli bacteria due to their inherent phagocytic activity. Functionalized sperm micromotors with intrinsic chemotactic motile behavior were reported. ${ }^{62}$ The activated sperm micromotors exhibited self-guided motion along a gradient of egg secretions toward the target area due to their chemotactic nature. The speed of the obtained sperm micromotors was regulated by decreasing osmolarity pressure in the surroundings that led to shortening of the flagellum length. A shorter flagellum beating in the surroundings would result in a smaller net displacement than sperm with normal flagellum length. Zhuang et al. ${ }^{63}$ developed a biohybrid MNM system propelled by multiple bacteria with a chemotactic response to L-serine (chemoattractant).

\section{Biosafety assessments of MNMs}

The review of biosafety assessments of MNMs is an underexplored area. However, the application of MNMs in the biomedical field demands a clear-cut understanding of their biosafety in vitro and in vivo. It is noticeable that the concept "biosafe" does not mean that the materials must be completely nontoxic and harmless. Make sure materials is safe to use and it is low toxicity, tolerable and can't cause a severe damage in the human body. To meet the biomedical application requirement, two main strategies 
including biodistribution and toxicity are explored to assess the biosafety issue of MNMs using cell and animal models.

Biodistribution of MNMs in organisms needs to be evaluated because foreign materials may accumulate in different organs and cause health hazards due to their potential toxicity. On the other hand, the study of biodistribution of MNMs is also important to realize their theranostic potential. Theranostics as an emerging concept which integrates therapy and diagnosis has become one of the hot investigations in the field of medical MNMs. After the biomedical MNMs enter the body through injection, oral administration, inhalation or other ways, it is expected to monitor these MNMs in organs and the targeted disease site. Thus, MNMs can be monitored noninvasively and provide people with more medical information through a single administration. Wang and coworkers ${ }^{79}$ evaluated the biodistribution of prepared biocompatible and biodegradable MNMs in tumor tissues and major organs via ex vivo imaging (as shown in Fig. 8A). The results showed that the autonomous propulsion of MNMs could greatly improve their local distribution and retention time in the targeted peritoneal cavity. The quantitative accumulation of payloads released from MNMs in tumor and major organs was studied. To be specific, the prepared MNMs exhibited better biodistribution and longer retention time in not only tumor but also major organs such as liver, kidney and colon. In general, the biodistribution of MNMs is highly dependent on their surface coverage and core diameter. Li et $a l .{ }^{37}$ studied the biodistribution and retention of MNMs with different enteric coating thickness in the stomach and several
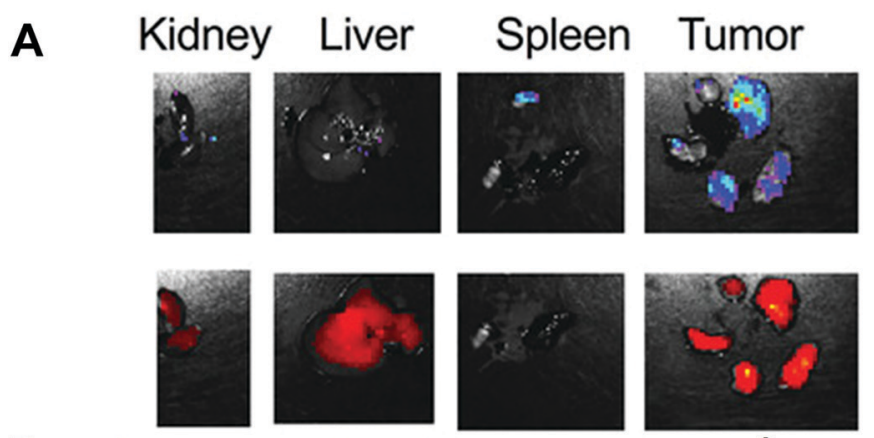

Colon

B
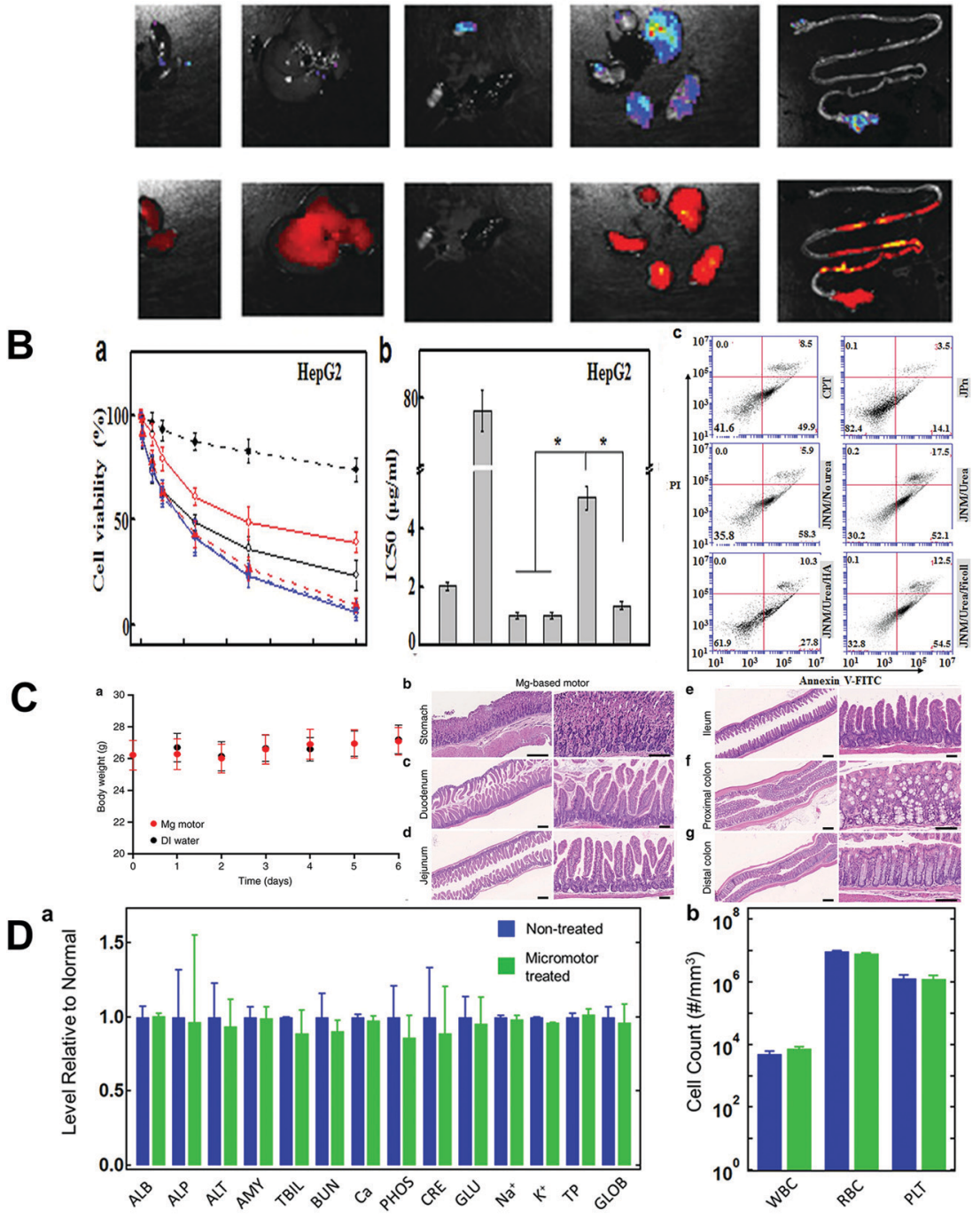

Fig. 8 Biosafety assessment of MNMs. (A) Representative IVIS images of organs at $24 \mathrm{~h}$ after micromotor treatment. (B) Cell viability, IC50 and flow cytometry analysis of HepG2 cells after different treatments. (C) Mouse body weight log from day 0 to day 6 of the toxicity study and histological staining of different sections for in vivo toxicity evaluation of $\mathrm{Mg}$-based micromotors. (D) Comprehensive blood chemistry panel taken from nontreated mice and mice treated for 30 days with Fe/Se-loaded micromotors. (A) Reproduced with permission. ${ }^{79}$ Copyright 2020, Wiley-VCH. (B) Reproduced with permission. ${ }^{80}$ Copyright 2019, Elsevier B.V. (C) Reproduced with permission. ${ }^{71}$ Copyright 2017, Springer Nature. (D) Reproduced with permission. ${ }^{81}$ Copyright 2019, American Chemical Society. 
segments of the gastrointestinal tract such as duodenum, jejunum and ileum. The results demonstrated that the coating thickness could greatly affect the biodistribution of MNMs in the gastrointestinal tract. In addition, cell-membrane coated MNMs or cell-based MNMs are expected to reduce/avoid biofouling and immune clearance and circulate in the body.

Researchers usually study the toxicity of MNMs via in vitro or in vivo assessments. In vitro assessment is usually conducted by cell cultivation. In in vitro experiments, the usually used cells include tumor cells (such as HepG2, H22, ${ }^{80}$ ID8-Defb29/VEGF$\mathrm{A},{ }^{79} \mathrm{~A} 549^{81}$ ), normal cells (ECs, human umbilical vein endothelial cells $^{82}$ ) and immune cells (macrophages ${ }^{79}$ ). The most commonly used method to evaluate the cytotoxicity of MNMs is assessing cell viability and proliferation. Lactate dehydrogenase analysis and the hemolysis test are conducted to investigate the cytotoxicity of MNMs on the stability of the cell membrane. Flow cytometry is used to study the changes in tumor cell apoptosis and cell cycle consequential to different treatments. Chen et al. ${ }^{80}$ explored the cytotoxicity of MNMs using HepG2 and H22 cell models and compared the IC50 values of different treatments (as depicted in Fig. 8B). Apoptosis induction of MNMs to cancer cells was analyzed with flow cytometry.

Before MNMs are applied in real clinical research, it is pivotal to investigate their biosafety issues in vivo. The in vivo assessments of MNMs' toxicity are much more complicated than in vitro experiments. Some physical signs such as body weight, tumor size and excrement analysis are necessary to investigate the toxicity of MNMs in vivo. In addition, the histological structures of main organs such as heart, liver, spleen, lung, kidney, thymus and other organs in mice are employed to assess the MNMs' toxicity. By means of routine HE staining, immunohistochemistry and electron microscopy, the histological characteristics and degrees of inflammation are observed. As indicated in Fig. 8C, the toxicity profile of biomedical MNMs for stomach infection was evaluated in the stomach and the lower gastrointestinal tract using a mouse model. ${ }^{71}$ Body weight changes of the mice were recorded as an aspect of the toxicity assessment. The stomach and lower gastrointestinal tract sections were stained with HE. Histopathology alteration, sign of inflammation and mouse body weight were evaluated to study the MNMs' toxicity in vivo. Karshalev et al. ${ }^{81}$ evaluated the toxicity of medical MNMs to blood composition, blood cell populations and tissue histology of major organs in the mouse (as shown in Fig. 8D). No evident difference was found in blood analytical indices and appearance of major organs between the MNM treatment group and the healthy mice group. In addition, the potential toxicity of MNMs can be further evaluated using TUNEL assay to study the level of cell apoptosis as an indicator of homeostasis in related tissues. ${ }^{82,83}$

However, the present toxicity assessment of MNMs based on cell and animal models is relatively rudimentary, and it can't evaluate the toxicity in a systematic and comprehensive way. Here, we make a non-exhaustive list of some biosafety issues which need to be addressed for application of MNMs in the healthcare field:

(1) The design of biosafe MNMs demands reasonable optimization of some essential features including shape, size, surface charge, composition and biofunctionalized decoration. On the other hand, it is necessary to investigate the relationship between physicochemical properties of MNMs and biosafety outcomes. ${ }^{84-86}$

(2) The biosafety of micro/nanoparticles is associated with different transport ways. In general, compared with other transport pathways, namely, ingestion, injection and skin absorption, micro/nanoparticle inhalation is regarded as the most hazardous route. ${ }^{87}$ When assessing the biosafety of MNMs, it is necessary to elucidate all the transport pathways and transport-related changes including the stability of MNMs in the organism.

(3) For the biodistribution study of MNMs, novel analytical methods are needed for quantitative determination of MNMs in different organs and tissues, as well as properties of certain MNMs such as surface free energy. ${ }^{88}$

(4) For in vitro assay of MNMs, some micro/nanoparticles might distort the MTT and MTS cell viability assay along with LDH release assay. ${ }^{89,90}$ Thus, additional alternative assays are recommended for certain kinds of MNMs. Multi-omics technology can be applied in the nanotoxicology study and provide information for the future study of molecular mechanisms. ${ }^{85,91,92}$

(5) For MNMs designed for long term biomedical usage, the potential genotoxicity needs to be ruled out using cell and animal models. Besides, a subchronic toxicity study is needed when MNMs showed no detected genotoxic effect. Also, the subchronic toxicity study has to include toxicokinetic analysis to examine the level of systemic MNM uptake. ${ }^{93}$

(6) As reported the cytotoxicity of micro/nanoparticles in both bacterial $^{94}$ and mammalian cells ${ }^{95}$ is mainly attributed to plasma membrane damage. However, the complex interaction between MNMs and the plasma membrane has made it difficult to translate the cytotoxicity results to properties of MNMs. Therefore, greater insights into the mechanisms of the interaction between MNMs and the cell plasma membrane might provide vital information about the cytotoxicity of MNMs and explain how the MNMs alter or disrupt the plasma membrane. ${ }^{96}$

(7) Some computational modes in terms of the relationships between nanostructures and their activity profiles need to be further improved to identify the characteristics which dictate the biosafety aspect of MNMs and meet the requirement of the biosafe design of MNMs. ${ }^{97-99}$

\section{Conclusions and outlook}

In this review, we outlined the recent progress in biosafe MNMs for biomedical applications. The biocompatibility, biodegradability, biosafe propulsion as well as biosafety assessment of MNMs in the past decade have been thoroughly reviewed. Tremendous progress has been made in the design and fabrication of MNMs with different functionalities for biomedical applications. However, before MNMs are used in real healthcare application, the biosafety of MNMs has to be evaluated in vitro and in vivo. One of the most important things of concern for biosafety is to fabricate MNMs with good biodegradability and biocompatibility. The biodegradability and biocompatibility of MNMs mainly depend on the properties of used materials. Different selection of 
materials as components of MNMs would endow them with different degrees of host response, biodegradation mechanisms and functionalities to satisfy different application requirements. Biodegradable and biocompatible MNMs with a focus on their building blocks have been reviewed. Furthermore, successful incorporation of biosafe materials into MNMs demands appropriate propulsion strategies. Some biosafe propulsion methods including biosafe fuel propulsion and fuel-free propulsion by various external stimuli including light, magnetic field, ultrasound or living organisms have been compared. By combining biosafe building blocks and appropriate propulsion strategies, these MNMs can achieve predetermined tasks in vitro and in vivo. To meet the biomedical application requirement, toxicity and biodistribution of MNMs are explored to assess biosafety issues in vitro and in vivo.

However, there are several challenges which should be addressed for the potential application of MNMs in the biomedical field. When designing biodegradable and biocompatible MNMs, two criteria should be considered: timeframe of MNMs' function as well as their host response. First, during the period of MNMs' function, the biodegradable frameworks of MNMs should not only define their shapes and sizes, but also provide a platform to integrate other functional components into MNMs for multifunctionality and propulsion. The desired functioning time of MNMs relies on their applications. Biodegradable MNMs should be fully decomposed at a suitable rate tolerable to the organism after completing their tasks. The second consideration is the biocompatibility of building blocks. A comprehensive understanding of the host response to materials used in MNMs is essential for designing new biocompatible MNMs. Besides, the biocompatibility of MNMs can be focused with respect to three aspects, which are cell, tissue and clinical perspectives. However, the related in vitro and in vivo reports on these three perspectives are still limited and further research including some related technical parameters and data is needed to better understand the biocompatibility.

In addition, the propulsion of biosafe MNMs needs to be benign to the physiological environment. Toxic fuels can be avoided and biofriendly external stimuli such as light, ultrasound, magnetic field or living organisms have advantages in biocompatibility and controlling the movement of MNMs. Researchers are expected to make further improvement of propulsion force generated by the MNMs in the biological environment, on-demand motion control, fully biodegradable and biocompatible designs, long lifetime, and enhanced tissue retention for imaging and therapeutic applications. The interplay between building blocks, propulsion mechanism, functions of MNMs and organisms can be integrated into the design of new biosafe MNMs to improve their performance.

An efficient approach is needed to investigate the biosafety assessment issues of MNMs. Animal models are the optimum standard to investigate the biodistribution and toxicity of MNMs. Besides, the relevant in vitro tissue and cell models can also be used for biosafety assessments. It is expected that the biosafety assessment will foster MNMs' biosafe design. The biosafety quality needs to be built into MNMs with the understanding of critical factors that cause toxicity and the ability to "design out" those related factors. Recently, some safe design methods including particle doping and surface coating have emerged and can be used in the fabrication of MNMs. An appropriate particle doping strategy is expected to change the inherent physicochemical properties of materials and reduce their toxicity. Surface coating provides an effective method to improve surface properties including hydrophilicity and solubility, so as to reduce the toxicity and improve the biosafety. The continued development of biosafe MNMs could pave a promising way to create biomedical MNMs for real health-care applications.

\section{Conflicts of interest}

The authors declare that they have no conflict of interest.

\section{Acknowledgements}

This study was funded by the National Key Research and Development Program of China (2016YFC0500305-02), the National Science Foundation of China (21972035), the Heilongjiang Science Foundation for Excellent Young Scholars (YQ2019E018), and the Thirteen five national key research and development projects (Grant No. 2016YFC0500307-07).

\section{References}

1 T. Xu, W. Gao, L. Xu, X. Zhang and S. Wang, Adv. Mater., 2017, 29, 1603250.

2 M. Guix, C. C. Mayorgamartinez and A. Merkoci, Chem. Rev., 2014, 114, 6285-6322.

3 J. Li, W. Gao, R. Dong, A. Pei, S. Sattayasamitsathit and J. Wang, Nat. Commun., 2014, 5, 5026.

4 J. Wu, S. Balasubramanian, D. Kagan, K. M. Manesh, S. Campuzano and J. Wang, Nat. Commun., 2010, 1, 36.

5 M. Safdar, S. U. Khan and J. Janis, Adv. Mater., 2018, 30, 1703660.

6 S. Lin, T. Yu, Z. Yu, X. Hu and D. Yin, Adv. Mater., 2018, 30, 1705691.

7 C. Li, C. Guo, V. Fitzpatrick, A. M. S. Ibrahim, M. J. Zwierstra, P. C. Hanna, A. Lechtig, A. Nazarian, S. J. Lin and D. L. Kaplan, Nat. Rev. Mater., 2020, 5, 61-81.

8 M. Razavi, M. Fathi, O. Savabi, D. Vashaee and L. Tayebi, Ann. Biomed. Eng., 2014, 42, 2537-2550.

9 Y. Yang, Q. He, L. Duan, Y. Cui and J. Li, Biomaterials, 2007, 28, 3083-3090.

10 Z. Wu, Y. Wu, W. He, X. Lin, J. Sun and Q. He, Angew. Chem., Int. Ed., 2013, 52, 7000-7003.

11 M. Xuan, J. Shao, X. Lin, L. Dai and Q. He, Colloids Surf., A, 2015, 482, 92-97.

12 N. Hu, L. Wang, W. Zhai, M. Sun, H. Xie, Z. Wu and Q. He, Macromol. Chem. Phys., 2018, 219, 1700540.

13 Z. Wu, X. Lin, X. Zou, J. Sun and Q. He, ACS Appl. Mater. Interfaces, 2015, 7, 250-255.

14 N. Sugai, Y. Morita and T. Komatsu, Chem. - Asian J., 2019, 14, 2953-2957. 
15 A. Penafrancesch, J. Giltinan and M. Sitti, Nat. Commun., 2019, 10, 3188.

16 L. S. Nair and C. T. Laurencin, Prog. Polym. Sci., 2007, 32, 762-798.

$17 \mathrm{~J}$. Middleton and A. J. Tipton, Biomaterials, 2000, 21, 2335-2346.

18 F. Xie, T. Zhang, P. Bryant, V. Kurusingal, J. M. Colwell and B. Laycock, Prog. Polym. Sci., 2019, 90, 211-268.

19 X. Wang, X. Qin, C. Hu, A. Terzopoulou, X. Chen, T. Huang, K. Maniuraweber, S. Pane and B. J. Nelson, Adv. Funct. Mater., 2018, 28, 1804107.

20 M. Liu, L. Liu, W. Gao, M. Su, Y. Ge, L. Shi, H. Zhang, B. Dong and C. Y. Li, Nanoscale, 2014, 6, 8601-8605.

21 M. Liu, Y. Sun, T. Wang, Z. Ye, H. Zhang, B. Dong and C. Y. Li, J. Mater. Chem. C, 2016, 4, 5945-5952.

22 L. Liu, Y. Dong, Y. Sun, M. Liu, Y. Su, H. Zhang and B. Dong, Nano Res., 2016, 9, 1310-1318.

23 T. Kroupa, S. Hermanova, C. C. Mayorgamartinez, F. Novotný, Z. Sofer and M. Pumera, Langmuir, 2019, 35, 10618-10624.

24 Y. Tu, F. Peng, A. A. M. Andre, Y. Men, M. Srinivas and D. A. Wilson, ACS Nano, 2017, 11, 1957-1963.

25 B. J. Toebes, F. Cao and D. A. Wilson, Nat. Commun., 2019, 10, 1-6.

26 J. Wang, B. J. Toebes, A. S. Plachokova, Q. Liu, D. Deng, J. A. Jansen, F. Yang and D. A. Wilson, Adv. Healthcare Mater., 2020, 1901710.

27 A. Sitt, J. Soukupova, D. Miller, D. Verdi, R. Zboril, H. Hess and J. Lahann, Small, 2016, 12, 1432-1439.

28 B. J. Toebes, L. K. E. A. Abdelmohsen and D. A. Wilson, Polym. Chem., 2018, 9, 3190-3194.

29 C. G. Fraga, Mol. Aspects Med., 2005, 26, 235-244.

30 Y. Chen, Z. Xu, C. Smith and J. Sankar, Acta Biomater., 2014, 10, 4561-4573.

31 D. M. Vasconcelos, S. G. Santos, M. Lamghari and M. A. Barbosa, Biomaterials, 2016, 84, 262-275.

32 W. Gao, A. Pei and J. Wang, ACS Nano, 2012, 6, 8432-8438.

33 W. Gao, R. Dong, S. Thamphiwatana, J. Li, W. Gao, L. Zhang and J. Wang, ACS Nano, 2015, 9, 117-123.

34 M. Zhou, T. Hou, J. Li, S. Yu, Z. Xu, M. Yin, J. Wang and X. Wang, ACS Nano, 2019, 13, 1324-1332.

35 S. Virtanen, Mater. Sci. Eng., B, 2011, 176, 1600-1608.

36 C. Chen, E. Karshalev, J. Li, F. Soto, R. Castillo, I. Campos, F. Mou, J. Guan and J. Wang, ACS Nano, 2016, 10, 10389-10396.

37 J. Li, S. Thamphiwatana, W. Liu, B. E. De Avila, P. Angsantikul, E. Sandraz, J. Wang, T. Xu, F. Soto and V. Ramez, ACS Nano, 2016, 10, 9536-9542.

38 J. Delezuk, D. E. Ramirezherrera, B. E. De Avila and J. Wang, Nanoscale, 2017, 9, 2195-2200.

39 M. A. Lopezramirez, F. Soto, C. Wang, R. Rueda, S. Shukla, C. Silvalopez, D. Kupor, D. A. Mcbride, J. K. Pokorski and A. Nourhani, Adv. Mater., 2020, 32, 1905740.

40 L. Wang, S. Wu, G. Cao, Y. Fan, N. Dunne and X. Li, J. Mater. Chem. B, 2019, 7, 7439-7459.

41 M. Guix, A. K. Meyer, B. Koch and O. G. Schmidt, Sci. Rep., 2016, 6, 21701.

42 J. R. Baylis, J. H. Yeon, M. H. Thomson, A. Kazerooni, X. Wang, A. E. S. John, E. B. Lim, D. Chien, A. Lee, J.
Q. Zhang, J. M. Piret, L. S. Machan, T. F. Burke, N. J. White and C. J. Kastrup, Sci. Adv., 2015, 1, 1-9.

43 H. Choi, B. W. Hwang, K. M. Park, K. S. Kim and S. K. Hahn, Part. Part. Syst. Charact., 2020, 37, 1900418.

44 M. Vert, Y. Doi, K. Hellwich, M. Hess, P. Hodge, P. Kubisa, M. Rinaudo and F. Schue, Pure Appl. Chem., 2012, 84, 377-410.

45 B. E. De Avila, C. Angell, F. Soto, M. A. Lopezramirez, D. F. Baez, S. Xie, J. Wang and Y. Chen, ACS Nano, 2016, 10, 4997-5005.

46 M. Luo, S. Li, J. Wan, C. Yang, B. Chen and J. Guan, Enhanced Propulsion of Urease-Powered Micromotors by Multilayered Assembly of Ureases on Janus Magnetic Microparticles, Langmuir, 2020, DOI: 10.1021/acs.langmuir. 9b03315.

47 Z. Xiao, J. Chen, S. Duan, X. Lv, J. Wang, X. Ma, J. Tang and W. Wang, Chem. Commun., 2020, 56, 4728-4731.

48 A. Molinerofernandez, L. Arruza, M. A. Lopez and A. Escarpa, Biosens. Bioelectron., 2020, 112156.

49 K. Yuan, V. de la Asuncion-Nadal, Y. Li, B. Jurado-Sanchez and A. Escarpa, Graphdyine micromotors in living biomedia, Chem. - Eur. J., 2020, DOI: 10.1002/chem.202001754.

50 V. M. Kadiri, C. Bussi, A. W. Holle, K. Son, H. Kwon, G. Schutz, M. G. Gutierrez and P. Fischer, Adv. Mater., 2020, e2001114.

51 B. E. De Avila, W. Gao, E. Karshalev, L. Zhang and J. Wang, Acc. Chem. Res., 2018, 51, 1901-1910.

52 S. Wang, X. Liu, Y. Wang, D. Xu, C. Liang, J. Guo and X. Ma, Nanoscale, 2019, 11, 14099-14112.

53 C. J. Hu, L. I. Zhang, S. Aryal, C. Cheung, R. H. Fang and L. Zhang, Proc. Natl. Acad. Sci. U. S. A., 2011, 108, 10980-10985.

54 C. J. Hu, R. H. Fang, J. A. Copp, B. T. Luk and L. Zhang, Nat. Nanotechnol., 2013, 8, 336-340.

55 C. J. Hu, R. H. Fang, B. T. Luk and L. Zhang, Nat. Nanotechnol., 2013, 8, 933-938.

56 Y. Chen, M. Chen, Y. Zhang, J. H. Lee, T. Escajadillo, H. Gong, R. H. Fang, W. Gao, V. Nizet and L. Zhang, Adv. Healthcare Mater., 2018, 7, 1701366.

57 J. Shao, M. Abdelghani, G. Shen, S. Cao, D. Williams and J. C. M. Van Hest, ACS Nano, 2018, 12, 4877-4885.

58 C. Gao, Z. Lin, D. Wang, Z. Wu, H. Xie and Q. He, ACS Appl. Mater. Interfaces, 2019, 11, 23392-23400.

59 J. Shao, M. Xuan, H. Zhang, X. Lin, Z. Wu and Q. He, Angew. Chem., Int. Ed., 2017, 56, 12935-12939.

60 Z. Wu, T. Li, J. Li, W. Gao, T. Xu, C. Christianson, W. Gao, M. Galarnyk, Q. He and L. Zhang, ACS Nano, 2014, 8, 12041-12048.

61 Z. Wu, B. E. De Avila, A. Martin, C. Christianson, W. Gao, S. Thamphiwatana, A. Escarpa, Q. He, L. Zhang and J. Wang, Nanoscale, 2015, 7, 13680-13686.

62 C. Chen, X. Chang, P. Angsantikul, J. Li, B. EstebanFernández de Ávila, E. Karshalev, W. Liu, F. Mou, S. He, R. Castillo, Y. Liang, J. Guan, L. Zhang and J. Wang, Adv. Biosyst., 2018, 2, 1700160.

63 J. Zhuang and M. Sitti, Sci. Rep., 2016, 6, 32135. 
64 X. Yan, Q. Zhou, M. Vincent, Y. Deng, J. Yu, J. Xu, T. Xu, T. Tang, L. Bian, Y. X. J. Wang, K. Kostarelos and L. Zhang, Sci. Rob., 2017, 2, eaaq1155.

65 H. Wang, M. G. Potroz, J. A. Jackman, B. Khezri, T. Maric, N. Cho and M. Pumera, Adv. Funct. Mater., 2017, 27, 1702338.

66 M. Sun, X. Fan, X. Meng, J. Song, W. Chen, L. Sun and H. Xie, Nanoscale, 2019, 11, 18382-18392.

67 W. Wang, W. Duan, S. Ahmed, T. E. Mallouk and A. Sen, Nano Today, 2013, 8, 531-554.

68 C. Chen, E. Karshalev, J. Guan and J. Wang, Small, 2018, 14, e1704252.

69 Y. Wu, X. Lin, Z. Wu, H. Mohwald and Q. He, ACS Appl. Mater. Interfaces, 2014, 6, 10476-10481.

70 T. Patino, N. Feinergracia, X. Arque, A. Miguellopez, A. Jannasch, T. Stumpp, E. Schaffer, L. Albertazzi and S. Sanchez, J. Am. Chem. Soc., 2018, 140, 7896-7903.

71 B. E. de Avila, P. Angsantikul, J. Li, M. Angel Lopez-Ramirez, D. E. Ramirez-Herrera, S. Thamphiwatana, C. Chen, J. Delezuk, R. Samakapiruk, V. Ramez, M. Obonyo, L. Zhang and J. Wang, Nat. Commun., 2017, 8, 272.

72 M. Wan, H. Chen, Q. Wang, Q. Niu, P. Xu, Y. Yu, T. Zhu, C. Mao and J. Shen, Nat. Commun., 2019, 10, 966.

73 D. Xu, Y. Wang, C. Liang, Y. You, S. Sanchez and X. Ma, Small, 2019, 1902464.

74 J. Palacci, S. Sacanna, A. P. Steinberg, D. J. Pine and P. M. Chaikin, Science, 2013, 339, 936-940.

75 C. Chen, F. Soto, E. Karshalev, J. Li and J. Wang, Adv. Funct. Mater., 2019, 29, 1806290.

76 B. Jang, E. Gutman, N. Stucki, B. F. Seitz, P. D. Wendelgarcia, T. H. Newton, J. Pokki, O. Ergeneman, S. Pane and Y. Or, Nano Lett., 2015, 15, 4829-4833.

77 T. Li, J. Li, K. I. Morozov, Z. Wu, T. Xu, I. Rozen, A. Leshansky, L. Li and J. Wang, Nano Lett., 2017, 17, 5092-5098.

78 D. Walker, B. T. Kasdorf, H. Jeong, O. Lieleg and P. Fischer, Enzymatically active biomimetic micropropellers for the penetration of mucin gels, Sci. Adv., 2015, 1, e1500501.

79 C. Wang, B. E. Fernández de Ávila, R. Mundaca-Uribe, M. A. Lopez-Ramirez, D. E. Ramírez-Herrera, S. Shukla, N. F. Steinmetz and J. Wang, Small, 2020, 16, 1907150.

80 Z. Chen, T. Xia, Z. Zhang, S. Xie, T. Wang and X. Li, Chem. Eng. J., 2019, 375, 122109.
81 E. Karshalev, Y. Zhang, B. E. De Avila, M. Beltrangastelum, Y. Chen, R. Mundacauribe, F. Zhang, B. Nguyen, Y. Tong and R. H. Fang, Nano Lett., 2019, 19, 7816-7826.

82 L. Zhao, Y. Liu, S. Xie, P. Ran, J. Wei, Q. Liu and X. Li, Chem. Eng. J., 2020, 382, 123041.

83 J. Li, P. Angsantikul, W. Liu, B. Esteban-Fernandez de Avila, S. Thamphiwatana, M. Xu, E. Sandraz, X. Wang, J. Delezuk, W. Gao, L. Zhang and J. Wang, Angew. Chem., Int. Ed., 2017, 56, 2156-2161.

84 A. E. Nel, T. Xia, L. Madler and N. Li, Science, 2006, 311, 622-627. 85 A. E. Nel, T. Xia, H. Meng, X. Wang, S. Lin, Z. Ji and H. Zhang, Acc. Chem. Res., 2013, 46, 607-621.

86 A. E. Nel, L. Madler, D. Velegol, T. Xia, E. M. V. Hoek, P. Somasundaran, F. Klaessig, V. Castranova and M. Thompson, Nat. Mater., 2009, 8, 543-557.

87 G. Oberdorster, E. Oberdorster and J. Oberdorster, Environ. Health Perspect., 2005, 113, 823-839.

88 S. Liu and T. Xia, Small, 2020, 16, e2000603.

89 A. L. Holder, R. Gothgoldstein, D. Lucas and C. P. Koshland, Chem. Res. Toxicol., 2012, 25, 1885-1892.

90 T. Xia, R. F. Hamilton, J. C. Bonner, E. D. Crandall, A. Elder, F. Fazlollahi, T. A. Girtsman, K. Kim, S. Mitra, S. A. Ntim, G. Orr, M. Tagmount, A. J. Taylor, D. Telesca, A. Tolic, C. D. Vulpe, A. J. Walker, X. Wang, F. A. Witzmann, N. Wu, Y. Xie, J. I. Zink, A. Nel and A. Holian, Environ. Health Perspect., 2013, 121, 683-690.

91 L. Cui, X. Wang, B. Sun, T. Xia and S. Hu, ACS Nano, 2019, 13, 13065-13082.

92 Y. Wu, W. Feng, R. Liu, T. Xia and S. Liu, ACS Nano, 2020, 14, 877-890.

93 H. C. Winkler, T. Notter, U. Meyer and H. Naegeli, J. Nanobiotechnol., 2018, 16, 51.

94 L. Lai, S. Li, J. Feng, P. Mei, Z. Ren, Y. Chang and Y. Liu, Langmuir, 2017, 33, 2378-2386.

95 T. Yu, A. Malugin and H. Ghandehari, ACS Nano, 2011, 5, 5717-5728.

96 A. M. Farnoud and S. Nazemidashtarjandi, Environ. Sci. Nano, 2019, 6, 13-40.

97 X. Wang, B. Sun, S. Liu and T. Xia, NanoImpact, 2017, 6, 99-108. 98 R. Liu, S. George, R. Rallo, H. Zhang, T. Xia, A. E. Nel, K. A. Bradley and Y. Cohen, Analyst, 2014, 139, 943-953.

99 R. Liu, H. H. Liu, Z. Ji, C. H. Chang, T. Xia, A. E. Nel and Y. Cohen, ACS Nano, 2015, 9, 9303-9313. 